\title{
ANÁLISE MORFOLÓGICA E INFERÊNCIAS ECOLÓGICAS DE GRÃOS DE PÓLEN E ESPOROS (ÚLTIMOS $~ 8.000$ ANOS) DA LAGOA DA FERRADURA, ARMAÇÃO DOS BÚZIOS, RJ, BRASIL
}

\author{
ALINE GONÇALVES DE FREITAS \& MARCELO DE ARAUJO CARVALHO \\ Laboratório de Paleoecologia Vegetal, Departamento de Geologia e Paleontologia, Museu Nacional, UFRJ, 20940-040, \\ Rio de Janeiro, RJ, Brasil.tuttyfreitas@gmail.com, mcarvalho@mn.ufrj.br
}

\begin{abstract}
MORPHOLOGICAL ANALYSES AND ECOLOGICAL INFERENCES OF POLLEN GRAINS AND SPORES (LAST 8.000 YEARS) OF THE FERRADURA LAGOON, ARMAÇÃO DOS BÚZIOS, RJ, BRAZIL. This work presents descriptions of spores

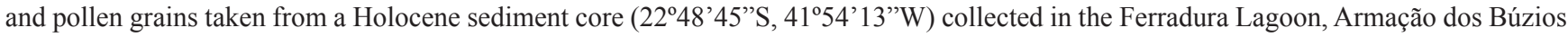
peninsula, Rio de Janeiro State, Brazil, during the last 8,060-7,770 ${ }^{14} \mathrm{C}$ cal. years BP. The chemical treatment of the sediments and palynological analyses made possible to identify, based on morphological criteria, 119 pollinic types. These types corresponded two families and two genera of bryophyte, six families and seven genera of pteridophyte, one genus of gymnosperm pollen (Podocarpus) and 107 types of angiosperms pollen that consisted of seven families and five genus of monocotyledoneae and 47 families and 66 genus of dicotyledoneae. The most common angiosperm types were: Fabaceae (20), Euphorbiaceae (7), Anacardiaceae (5), Malvaceae (5), Bignoniaceae (4), Sapindaceae (3), and Amaranthaceae (3). The most abundant taxa were: Actinostemon, Allagoptera, Anacardiaceae, Asteraceae, Azolla, Chenopodium, Cyathea, Euphorbiaceae, Myrtaceae, Poaceae, Schinus, Trema, cf. Trichilia and Typha. The palynoflora in this study are associated to restinga and semideciduous forests, demonstrating the potential of this material to provide environmental information relevant to paleoenvironmental reconstruction of the study area.
\end{abstract}

Key words: pollen morphology, pollen grains, cryptogam spores, paleovegetation, Holocene, Ferradura lagoon.

RESUMO - Este trabalho apresenta as descrições dos grãos de pólen e esporos extraídos de sedimentos do testemunho de sondagem RJ 92-5

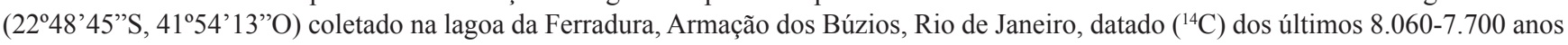
cal AP. O tratamento químico dos sedimentos, as análises palinológicas possibilitaram identificar, com base em critérios morfológicos, 119 tipos esporo-polínicos. Estes tipos correspondem a duas famílias e dois gêneros de briófitas, seis famílias e sete gêneros de pteridófitas, um gênero de gimnosperma (Podocarpus) e 107 tipos de grãos de pólen de angiospermas, sendo sete famílias e cinco gêneros de monocotiledôneas e 47 famílias e 76 gêneros de dicotiledôneas. Os táxons de angiospermas mais representativos foram: Fabaceae (20), Euphorbiaceae (8), Anacardiaceae (5), Malvaceae (5), Bignoniaceae (4), Sapindaceae (3), e Amaranthaceae (3). Os táxons mais abundantes foram: Actinostemon, Allagoptera, Anacardiaceae, Asteraceae, Azolla, Chenopodium, Cyathea, Euphorbiaceae, Myrtaceae, Poaceae, Schinus, Trema, cf. Trichilia e Typha. A palinoflora identificada neste trabalho relaciona-se à vegetação de restinga e floresta estacional semidecidual, demonstrando o potencial deste material em oferecer informações ambientais importantes para a reconstituição paleoambiental da região de estudo.

Palavras-chave: morfologia polínica, grãos de pólen, esporos de criptógamas, paleovegetação, Holoceno, lagoa da Ferradura.

\section{INTRODUÇÃO}

As análises palinológicas quaternárias constituem valiosa ferramenta para interpretações paleoambientais. Análises quantitativas das associações polínicas em depósitos holocenos fornecem a base para interpretação da comunidade vegetal em resposta às mudanças ambientais climáticas e antropogênicas (Kneller \& Peteet, 1999; Willard et al., 2004). As descrições morfológicas são fundamentais para a pesquisa palinológica do Quaternário, uma vez que os grãos de pólen e esporos subfósseis são morfologicamente similares aos produzidos pelas plantas atuais e permitem uma analogia com os táxons de famílias e gêneros botânicos além de permitir inferências mais seguras sobre a paleovegetação, e consequentemente sobre o paleoclima estudado.
A região de Cabo Frio, na qual está inserido o município de Armação dos Búzios, exibe uma diversidade vegetal que está diretamente relacionada com as variadas condições geomorfológicas e às condições climáticas locais (Araújo, 1997, 2000). A região abriga um dos doze Centros de Diversidade Vegetal do Brasil onde se preserva uma das poucas reservas naturais de pau-brasil (Caesalpinia echinata Lam.). A vegetação de Cabo Frio foi classificada por Ururahy et al. (1987) como Floresta Estacional Semidecidual ou mata seca (ou xerofítica), a qual possui similaridades com a caatinga nordestina. Segundo Ab'Saber (1977), a cobertura vegetal atual é remanescente de uma vegetação existente durante os períodos glaciais pleistocenos, mais secos e mais frios.

Embora os registros palinológicos quaternários do sul e 
sudeste brasileiros ainda representem análises pontuais, eles fornecem dados sobre a composição vegetacional e sobre as mudanças paleoambientais pleistocênicas (Behling et al., 2002; Freitas, 2005; Freitas et al., 2007) e holocênicas (Neves \& Lorscheitter, 1991; Cordeiro \& Lorscheitter, 1994; Luz \& Barth, 2000; Luz et al., 2006; Medeanic et al., 2006; Toledo et al., 2009). Em estudos polínicos da lagoa de Cima, Campos, RJ, Luz \& Barth $(2000,2002)$ registraram grãos de pólen de angiospermas indicadores de floresta atlântica, restinga, vegetação herbácea, pantanosa e de margens de lagos, em sedimentos datados dos últimos 6.100 anos. Análises palinológicas de testemunhos lacustres no norte fluminense revelaram sucessões vegetacionais entre a floresta úmida e a vegetação de campo durante o Holoceno (Luz et al., 2011). Essas mudanças foram provavelmente controladas pelas oscilações relativas do nível do mar na região. O rebaixamento relativo do nível de mar e a expansão da planície litorânea cerca de 6.500 e. 4.000 anos A.P. permitiu a colonização e expansão das plantas herbáceas e heliófitas (Luz et al., 2011).

Em investigação palinológica de um testemunho da bacia de Campos, de idade Pleistoceno-Holoceno, Freitas (2005) e Freitas et al. (2007) documentaram palinomorfos relacionados a esporos de briófitas e de pteridófitas, algas, grãos de pólen de gimnospermas e de angiospermas, esporos e frutificações de fungos. As variações nos percentuais dos palinomorfos continentais analisados refletem as mudanças paleoclimáticas, associadas às fases climáticas glaciais e interglaciais do Pleistoceno-Holoceno.

As análises esporo-polínicas de caráter morfológico e taxonômico, realizadas em sedimentos holocênicos, são fundamentais para uma interpretação mais segura da vegetação e, consequentemente para inferir sobre as mudanças climáticas e ambientais. Os tipos polínicos recuperados nos sedimentos da lagoa da Ferradura (Figura 1) foram identificados e descritos morfologicamente neste trabalho.

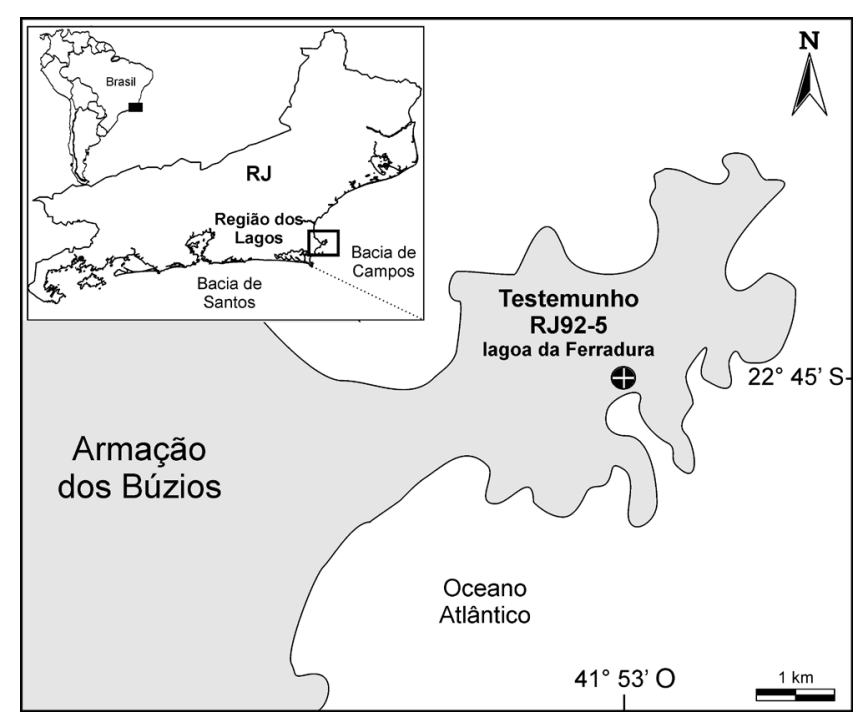

Figura 1. Localização do testemunho RJ 92-5, lagoa da Ferradura, Armação dos Búzios, Região dos Lagos, RJ, Brasil.

Figure 1. Location map of core RJ 92-5, Ferradura lagoon, Armação dos Búzios, Lakes Region, RJ, Brazil.

\section{Clima e vegetação atual}

O município de Armação dos Búzios situa-se na província geomorfológica de baixada litorânea tropical úmida, todavia apresenta clima semi-árido quente, com temperatura média anual de $25^{\circ} \mathrm{C}$, fortemente influenciado pela ressurgência e por correntes oceânicas (Martin et al., 1996; Tasayaco-Ortega, 1996). A umidade relativa do ar é alta devido à grande exposição aos ventos úmidos e as precipitações pluviométricas atingem $800 \mathrm{~mm} /$ ano com menos de $80 \mathrm{~mm} / \mathrm{mês}$ (FEEMA, 1988).

A vegetação da região de Cabo Frio, na qual está inserido o município de Armação dos Búzios, compreende floresta seca ou floresta estacional semidecidual, similar a caatinga nordestina (Ururahy et al., 1987; IBGE, 1992). Tais semelhanças estão relacionadas à história geomorfológica e climática regional (Dantas et al., 2009). Os levantamentos florísticos e fitossociológicos (Farág, 1999; Araújo, 2000; Lima, 2000; Fernandes \& Sá, 2000; Sá, 2002, 2006; Kurtz et al., 2009) realizados em Armação dos Búzios apontam as formações de floresta estacional semidecidual ou mata seca de encosta e restinga, como sendo os tipos vegetacionais dominantes na região. O município apresenta uma diversidade de ambientes. Em associação às suas províncias geomorfológicas de colinas, tabuleiros e morros costeiros, planície litorânea, planície aluvial e áreas alagadas (Dantas et al., 2009), são registradas vegetação de manguezal, formações herbáceas salinas e dulcícolas, formações arbustivas xeromórficas, formações florestais e restinga (Araújo, 2000).

As espécies marcantes da flora de Cabo Frio e Armação dos Búzios são o cacto endêmico Pilosocereus ulei (K.Schum.) Byles \& G.D.Rowley, característico de vegetação xerofítica, as leguminosas Bauhinia albicans Vogel e Machaerium leucopterum Vogel (Lima, 2000) e a liana Skytanthus hancorniifolius Miers (Apocynaceae), que exibem uma distribuição a parte da caatinga nordestina, sugerindo antigas ligações entre estas duas regiões durante o período mais seco do Pleistoceno (Ab'Saber, 1977; Araujo, 1997).

\section{MATERIAL E MÉTODOS}

O testemunho RJ 92-5 (22 $\left.48^{\prime} 45^{\prime \prime} \mathrm{S}, 4^{\circ} 54^{\prime} 13^{\prime \prime} \mathrm{O}\right)$ foi coletado por meio de vibro-testemunhador, na lagoa da Ferradura, em Armação dos Búzios, Região dos Lagos, RJ (Figura 1). Seus sedimentos compreendem argilas orgânicas intercaladas a lâminas carbonáticas, argila arenosa nos níveis 80 e $100 \mathrm{~cm}$ e areia fina argilosa na base. Registra-se a presença de microgastróspodes (Heleobia australis: 5, 52, 122 e $316 \mathrm{~cm}$ ), ostracodes (Cyprideis sp. a $122 \mathrm{~cm}$, C. salebrosa e Perissocytheridea sp. a $52 \mathrm{~cm}$ ) e carófitas Girogonites sp. a $52 \mathrm{~cm}$ ) nos níveis de argilas orgânicas (J. Monteiro e C. Bergue, com. pes.).

A deposição holocênica dos sedimentos da lagoa da Ferradura iniciou antes de 8.060-7.770 anos cal AP e seguiu até 160-10 anos cal AP, de acordo com as datações radiocarbônicas ${ }^{14} \mathrm{C}$ realizadas no Laboratório Beta Analytic Inc., EUA (Tabela 1). As idades convencionais foram calibradas de acordo com a curva IntCal04 (Reimer et al., 2004) com precisão de 2 sigma, usando o programa CALIB Rev 5.0.1 (Stuiver \& Reimer, 1993). 
Foram analisadas 49 amostras de sedimentos (ca. $2 \mathrm{~g})$ do testemunho RJ 92-5. O tratamento químico seguiu o método palinológico padrão para sedimentos quaternários (Ybert et al., 1992) usando $\mathrm{HCl}$ a $10 \%$ e $\mathrm{HF}$ a $40 \%$ para eliminar a fração mineral, acetólise e líquido denso $\left(\mathrm{ZnCl}_{2}, \mathrm{D}=2\right)$ para a concentração da matéria orgânica.

As descrições dos esporos de briófitas e pteridófitas se fundamentaram nos caracteres diagnósticos de cicatriz, forma, ornamentação da perina e da exina e tamanho dos grãos. Os grãos de pólen de gimnospermas e angiospermas foram descritos segundo os critérios de tamanho dos grãos, unidade de dispersão polínica, polaridade, simetria, forma, número, posição e caráter das aberturas, e ornamentação da exina (Barth \& Melhem, 1988; Punt et al., 2007) (Apêndice 1). As fotomicrografias foram efetuadas no programa Axion Vision acoplado ao microscópio de luz transmitida Zeiss Axioplan, em aumentos de 400x e 1000x (Figuras 2-7).

Para as identificações e descrições polínicas foram consultados catálogos polínicos das restingas do Rio de Janeiro e literatura especializada como: (Erdtman, 1952, 1965; Barth \& Bouzada, 1964; Barth, 1964, 1972a,b; Barth \& Silva, 1965; Barth \& Yoneshigue, 1966; Heusser, 1971; Barth \& Barbosa, 1972a,b; Salgado-Labouriau, 1973; Barth et al., 1976, 1992, 1998; Müller \& Caratini, 1977; Ybert, 1979; Gonçalves-Esteves et al., 1986, 1992, 2007; Lorscheitter, 1989; Roubik \& Moreno, 1991; GonçalvesEsteves \& Crespo, 1994a,b; Gonçalves-Esteves \& Ferreira, 1994a,b; Sylvestre, 1995; Carreira et al., 1996; Scheel et al., 1996; Lorscheitter et al., 1999, 2002, 2005; GonçalvesEsteves \& Cattete, 1997; Barth \& Justo, 2000; Luz \& Barth, 2000; Mendonça \& Gonçalves-Esteves, 2000a,b; GonçalvesEsteves \& Mendonça, 2001; Milward-de-Azevedo et al., 2004; Moreira et al., 2004; Souza et al., 2004a,b; Willard et al., 2004; Freitas, 2005; Cruz-Barros et al., 2006; Freitas et al., 2007), assim como a coleção de referência das plantas vasculares da região de Cabo Frio, RJ, que está depositada no Laboratório de Paleoecologia Vegetal do Departamento de Geologia e Paleontologia do Museu Nacional.

Os tipos esporo-polínicos foram identificados em níveis taxonômicos de família e gênero botânicos (Apêndice 1). O tipo esporo-polínico caracteriza-se por estabelecer uma proximidade entre o material analisado e determinado grupo taxonômico (Salgado-Labouriau, 1973; Lorscheitter,

Tabela 1. Datações radiocarbônicas $A M S{ }^{14} \mathrm{C}$ realizadas em sedimentos orgânicos totais do testemunho RJ 92-5.

Table 1. Radiocarbon AMS ${ }^{14} \mathrm{C}$ dates accomplished in total organic sediments of core RJ 92-5.

\begin{tabular}{ccccc}
\hline Prof.(cm) & $\begin{array}{c}\text { Código do } \\
\text { Laboratório }\end{array}$ & $\begin{array}{c}\text { Idades } \\
\text { AMS }{ }^{14} \mathrm{C} \\
\text { convencionais } \\
\text { (anos AP) }\end{array}$ & $\begin{array}{c}\text { Idades calibradas } \\
\text { cal AP } \\
\text { (IntCa104 2 sigma) }\end{array}$ & ${ }^{13} \mathrm{C}$ \\
\hline 2.0 & Beta-100116 & $100 \pm 50$ & $160-10$ & -25.2 \\
60.0 & Beta-270654 & $3.960 \pm 40$ & $4.530-4.290$ & -22.6 \\
150.0 & Beta-100112 & $4.830 \pm 80$ & $5.730-5.450$ & -20.8 \\
169.0 & Beta-100113 & $4.910 \pm 80$ & $5.780-5.580$ & -18.3 \\
176.5 & Beta-100114 & $4.900 \pm 100$ & $5.900-5.470$ & -23.0 \\
341.0 & Beta-100115 & $7.090 \pm 100$ & $8.060-7.700$ & -26.6 \\
\hline
\end{tabular}

1989). As descrições polínicas obedecem à ordem alfabética de família botânica e correspondem às atualizações da Angiosperm Phylogeny Group III (APG III, 2003) (Apêndice 1). Os tipos esporo-polínicos holocênicos identificados foram relacionados aos ecossistemas atuais de floresta ombrófila densa, floresta estacional semidecidual, elementos da transição entre a floresta e a restinga, restinga, manguezal, herbáceas, higrófitas, pteridófitas e briófitas (Lorenzi, 1998, 2002, 2009; Costa \& Dias, 2001; Souza \& Lorenzi, 2008; Dantas et al., 2009; Kurtz et al., 2009) (Tabela 2).

\section{RESULTADOS E DISCUSSÃO}

\section{Análises palinológicas}

Foram determinados e descritos 119 tipos esporo-polínicos relacionados a esporos e grãos de pólen (Figuras 2-7; Tabela 2, Apêndice 1), os quais se distribuem em: duas famílias de briófitas sensu lato (Phaeoceros e Sphagnum), seis famílias de pteridófitas com sete gêneros (Azolla, Asplenium, Blechnum, Cyathea, Polypodium, Acrostichum, Pityrogramma), uma família de gimnosperma com um gênero (Podocarpus) e 107 tipos de grãos de pólen de angiospermas, sendo sete famílias e cinco gêneros de monocotiledôneas (Sagittaria, Allagoptera, Bromeliaceae, cf. Bulbostylis, Poaceae, Ruppia e Typha) e 47 famílias e 66 gêneros de dicotiledôneas. As famílias de angiospermas mais representativas em números de tipos polínicos foram: Fabaceae (20), Euphorbiaceae (8), Anacardiaceae (5), Malvaceae (5), Bignoniaceae (4), Sapindaceae (3), e Amaranthaceae (3).

Os tipos polínicos descritos (Figuras 2-7) são apresentados no Apêndice 1. Os tipos esporo-polínicos identificados relacionamse com os levantamentos florísticos e fitossociológicos de floresta estacional semidecidual (Kurtz et al., 2009) e mata de restinga (Fernandes \& Sá, 2000; Lobão \& Kurtz, 2000; Sá, 2002; Fonseca-Kruel et al., 2009) realizados na Região dos Lagos, RJ.

\section{CONSIDERAÇÕES FINAIS}

O testemunho RJ 92-5, coletado na lagoa da Ferradura, representa um dos registros mais completos da sedimentação holocênica em áreas costeiras brasileiras. Seus sedimentos datam desde antes de 8.060-7.770 ${ }^{14} \mathrm{C}$ anos cal. AP até o presente.

Os tipos esporo-polínicos recuperados dos sedimentos lacustres-lagunares, morfologicamente descritos e determinados taxonomicamente, constituem um registro pioneiro para a Região dos Lagos fluminenses. A assembleia esporo-polínica relaciona-se com as fitofisionomias atuais de floresta estacional semidecidual, restinga e manguezal. Os tipos identificados exibem diversidade e abundância, e representam vegetação arbustivo-arbórea e herbácea.

As análises morfológicas dos distintos grãos de pólen de gimnospermas, monocotiledôneas, dicotiledôneas e esporos de briófitas e pteridófitas demonstraram um grande potencial para a caracterização de paleoambiente, pois, além de fornecerem informações sobre a dinâmica da vegetação, foi possível verificar a evolução da paleolaguna, a partir de 7.940 anos AP e sua colmatação em paleolagoa, após 2.810 anos AP até o recente. 
Tabela 2. Tipos palinológicos identificados no testemunho RJ 92-5 e os ecossistemas relacionados (Lorenzi, 1998, 2002, 2009; Costa \& Dias, 2001; Souza \& Lorenzi, 2008; Dantas et al., 2009; Kurtz et al., 2009). Abreviaturas: C, Caesalpinoideae; Fabaceae P, Papilionoideae e M, Mimosoideae. [*] Ocorrentes na floresta estacional semidecidual de Armação dos Búzios, RJ.

Table 2. Pollen types identified in core RJ 92-5 and the related ecosystems (Lorenzi, 1998, 2002, 2009; Costa \& Dias, 2001; Souza \& Lorenzi, 2008; Dantas et al., 2009; Kurtz et al., 2009). Abbreviations: C, Caesalpinoideae; Fabaceae P, Papilionoideae and M, Mimosoideae. [] Ocurring in semideciduous forest of Armação dos Búzios, RJ.

\begin{tabular}{|c|c|c|}
\hline FLORESTA & Fabaceae M Mimosa & Araliaceae cf. Hydrocotyle \\
\hline Podocarpaceae Podocarpus & Fabaceae M Piptadenia & Convolvulaceae Tipo Convolvulaceae \\
\hline Fabaceae C Copaifera & Fabaceae P Zollernia & Fabaceae P Canavalia \\
\hline Anacardiaceae Astronium* & Fabaceae P Pterocarpus & Lythraceae Cuphea \\
\hline Anacardiaceae Spondias* & Fabaceae P Tipo Fabaceae P & Malpighiaceae Byrsonima \\
\hline Fabaceae C Caesalpinia* & Malpighiaceae Tipo Malpighiaceae & Malvaceae Hibiscus \\
\hline Araliaceae cf. Dendropanax & Malvaceae Pseudobombax & Myrtaceae cf. Psidium \\
\hline Phyllanthaceae Hyeronima & Myrtaceae Tipo Myrtaceae & Rhamnaceae Tipo Rhamnaceae \\
\hline Euphorbiaceae Actinostemon* & Meliaceae cf. Trichilia & Verbenaceae cf. Lantana \\
\hline Combretaceae Tipo Combretaceae & Passifloraceae Passiflora & \\
\hline Euphorbiaceae Pachystroma* & Polygalaceae Polygala & MANGUEZAL \\
\hline Fabaceae C cf. Melanoxylon & Malvaceae cf. Waltheria & Acanthaceae Avicennia \\
\hline Fabaceae C Peltogyne* & Rubiaceae Psychotria & Combretaceae cf. Laguncularia \\
\hline Fabaceae C Pterogyne* & Sapindaceae Serjania & Rhizophoraceae Rhizophora \\
\hline \multicolumn{3}{|l|}{ Fabaceae M Anadenanthera* } \\
\hline Fabaceae P Acosmium* & RESTINGA & HERBÁCEAS \\
\hline Fabaceae P Tipo Dalbergia-Machaerium & Bignoniaceae cf. Adenocalymma & Amaranthaceae Chenopodium \\
\hline Fabaceae P Tipo Lonchocarpus-Ormosia & Boraginaceae cf. Cordia & Asteraceae Tribo Eupatorieae \\
\hline Fabaceae P Swartzia & Connaraceae Connarus & Asteraceae Tribo Vernonieae \\
\hline Fabaceae P Platymiscium* & Euphorbiaceae Dalechampia & Poaceae Tipo Poaceae \\
\hline Malvaceae Ceiba* & Loranthaceae Struthanthus & Rubiaceae Borreria \\
\hline Meliaceae cf. Cedrela* & Malvaceae Abutilon & \\
\hline \multirow[t]{2}{*}{ Phyllanthaceae Tipo Phyllanthaceae } & Acanthaceae Justicia & AQUÁTICAS \\
\hline & Sapindaceae Allophylus & Typhaceae Typha \\
\hline FLORESTA + RESTINGA & Solanaceae Solanum & Ruppiaceae Ruppia \\
\hline Anacardiaceae Tipo Anacardiaceae & Lamiaceae cf. Aegiphila & Cyperaceae cf. Bulbostylis \\
\hline Apocynaceae Tipo Apocynaceae & Amaranthaceae Alternanthera & Onagraceae Ludwigia \\
\hline Arecaceae Allagoptera & Anacardiaceae Schinus & Lentibulariaceae Utricularia \\
\hline Bignoniaceae Tipo Bignoniaceae & Aquifoliaceae Ilex & Polygonaceae Polygonum \\
\hline Bignoniaceae cf. Arrabidaea & Brassicaceae Capparis & \\
\hline Bignoniaceae Tabebuia & Celastraceae cf. Maytenus & PTERIDÓFITAS \\
\hline Bromeliaceae Tipo Bromeliaceae & Clusiaceae cf. Clusia & Azollaceae Azolla \\
\hline Cactaceae Tipo Cactaceae & Clusiaceae cf. Garcinia & Aspleniaceae Asplenium \\
\hline Cannabaceae Celtis & Ericaceae Tipo Ericaceae & Blechnaceae Blechnum \\
\hline Cannabaceae Trema & Urticaceae cf. Cecropia & Cyatheaceae Cyathea \\
\hline Elaeocarpaceae Sloanea & Moraceae cf. Ficus & Polypodiaceae Polypodium \\
\hline Erythroxylaceae Erythroxylum & Myrsinaceae Rapanea & Pteridaceae Acrostichum \\
\hline Tipo Euphorbiaceae & Ochnaceae Ouratea & Pteridaceae Pityrogramma \\
\hline Euphorbiaceae Alchornea & Polygonaceae Coccoloba & \\
\hline Euphorbiaceae Croton & Rutaceae Zanthoxylum & BRIÓFITAS \\
\hline Euphorbiaceae Sapium & Sapindaceae Paullinia & Anthocerotaceae Phaeoceros \\
\hline Euphorbiaceae cf. Sebastiania & Sapotaceae cf. Syderoxylon & Sphagnaceae Sphagnum \\
\hline Fabaceae C Tipo Fabaceae C & Amaranthaceae Tipo Gomphrena & \\
\hline Fabaceae C Bauhinia & Anacardiaceae Tapirira & \\
\hline
\end{tabular}


A influência da salinidade, em virtude das variações relativas do nível local do mar foi atestada através do registro de vegetação herbácea halófita (Chenopodium e Ruppia) e de manguezal (Rizhophora, Avicennia, Laguncularia e Acrostichum), entre 7.940-2.810 anos AP, com expressividade entre 7.940-5.750 anos AP, passando a vegetação herbácea aquática característica de brejos e ambientes estagnados, como margens de lagoas (Poaceae, Typha, Polypodium e Cyathea) após 2.810 anos AP. A vegetação arbustivo-arbórea de restinga e floresta estacional semidecidual esteve presente ao longo dos últimos $\sim 8.000$ anos na região estudada.

Os dados palinológicos corroboram as curvas de variação do nível do mar holocênico propostas para a região estudada.

\section{AGRADECIMENTOS}

Os autores agradecem a J.-P. Ybert (DGP/Museu Nacional), L. Martin (ORSTOM) e R. Scheel-Ybert (Antropologia/
Museu Nacional) pela coleta do testemunho analisado; a V. Gonçalves-Esteves (Botânica/Museu Nacional) pelas discussões palinológicas; a G. Bianchini (Antropologia/ Museu Nacional) pelas discussões botânicas e ecológicas; a S. Agostinho (Geologia/UFPE) pelo auxílio na formatação final deste manuscrito; a H. Mort (Geologia/UFPE) pelas sugestões no idioma inglês. Aos revisores anônimos deste manuscrito e ao CNPq (141161/2007-8) pela bolsa do doutorado concedida à primeira autora.

\section{REFERÊNCIAS}

Ab'Saber, A.N. 1977. Espaços ocupados pela expansão dos climas secos na América do Sul por ocasião dos períodos glaciais quaternários. Paleoclimas, 3:1-19.

APG III. Angiosperms Phylogeny Group III Classification. 2009. An update of Angiosperm Phylogeny Group Classification for the orders and families of flowering plants: APG III. Botanical Journal of the Linnean Society, 161:105-121. doi:10.1111/j.10958339.2009.00996.x
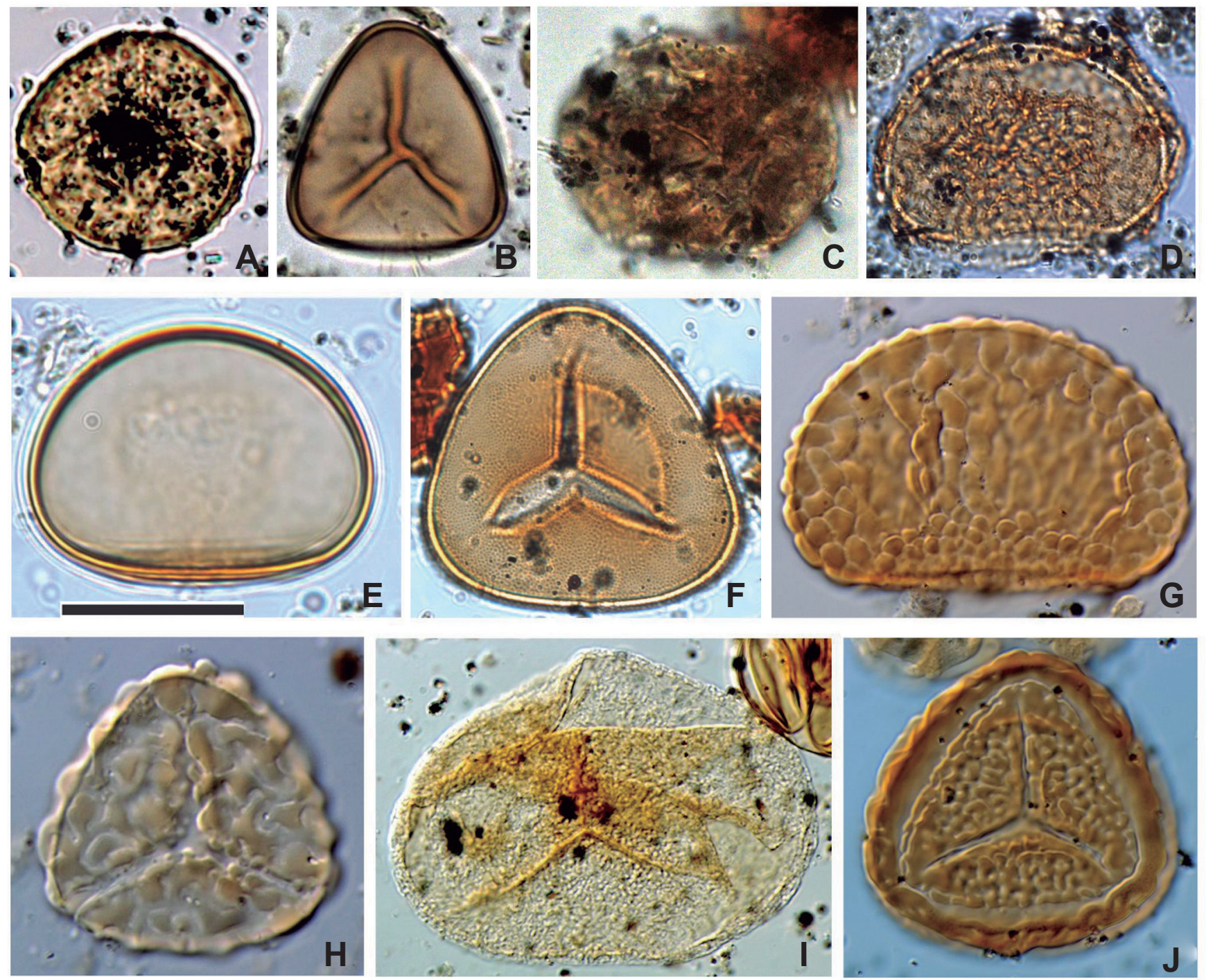

Figura 2. Fotomicrografias de esporos de briófitas e pteridófitas do testemunho RJ 92-5. A, Tipo/Type 1 Phaeoceros; B, Tipo/Type 2 Sphagnum; C, Tipo/Type 3 Azolla; D, Tipo/Type 4 Asplenium; E, Tipo/Type 5 Blechnum; F, Tipo/Type 6 Cyathea; G, Tipo/Type 7 Polypodium monolete; H, Tipo/Type 8 Polypodium trilete; I, Tipo/Type 9 Acrostichum; J, Tipo/Type 10 Pityrogramma. Escala = $20 \mu \mathrm{m}$.

Figure 2. Photomicrographs of bryophytes and pteridophytes spores of core RJ 92-5. Scale bar $=20 \mu \mathrm{m}$. 

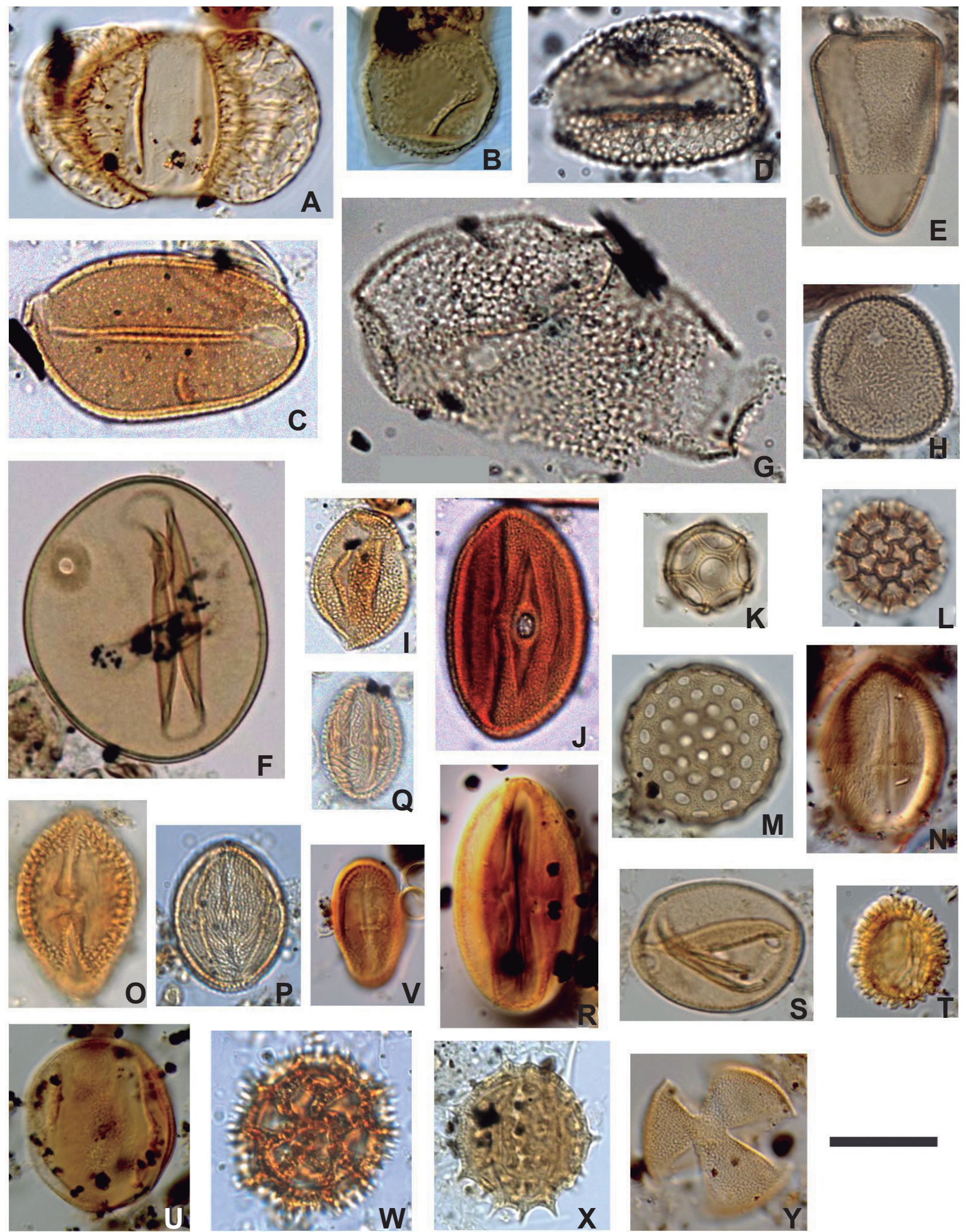

Figura 3. Fotomicrografias de grãos de pólen de gimnospermas e angiospermas do testemunho RJ 92-5. A, Tipo/Type 11 Podocarpus; B, Tipo/ Type 12 Sagittaria; C, Tipo/Type 13 Allagoptera; D, Tipo/Type 14 Bromeliaceae; E, Tipo/Type 15 cf. Bulbostylis; F, Tipo/Type 16 Poaceae; G, Tipo/Type 17 Ruppia; H, Tipo/Type 18 Typha; I, Tipo/Type 19 Avicennia; J, Tipo/Type 20 Justicia; K, Tipo/Type 21 Alternanthera; L, Tipo/Type 22 Gomphrena; M, Tipo/Type 23 Chenopodium; N, Tipo/Type 24 Anacardiaceae; O, Tipo/Type 25 Astronium; P, Tipo/Type 26 Schinus; Q, Tipo/ Type 27 Tapirira; R, Tipo/Type 28 Spondias; S, Tipo/Type 29 Apocynaceae; T, Tipo/Type 30 Ilex; U, Tipo/Type 31 cf. Dendropanax; V, Tipo/ Type 32 Hydrocotyle; W, Tipo/Type 33 Tribo Vernonineae; X, Tipo/Type 34 Tribo Eupatorieae; Y, Tipo/Type 35 Bignoniaceae. Escala = $20 \mu$ m.

Figure 3. Photomicrographs of gymnosperms and angiosperms pollen grains of core RJ 92-5. Scale bar $=20 \mu \mathrm{m}$. 

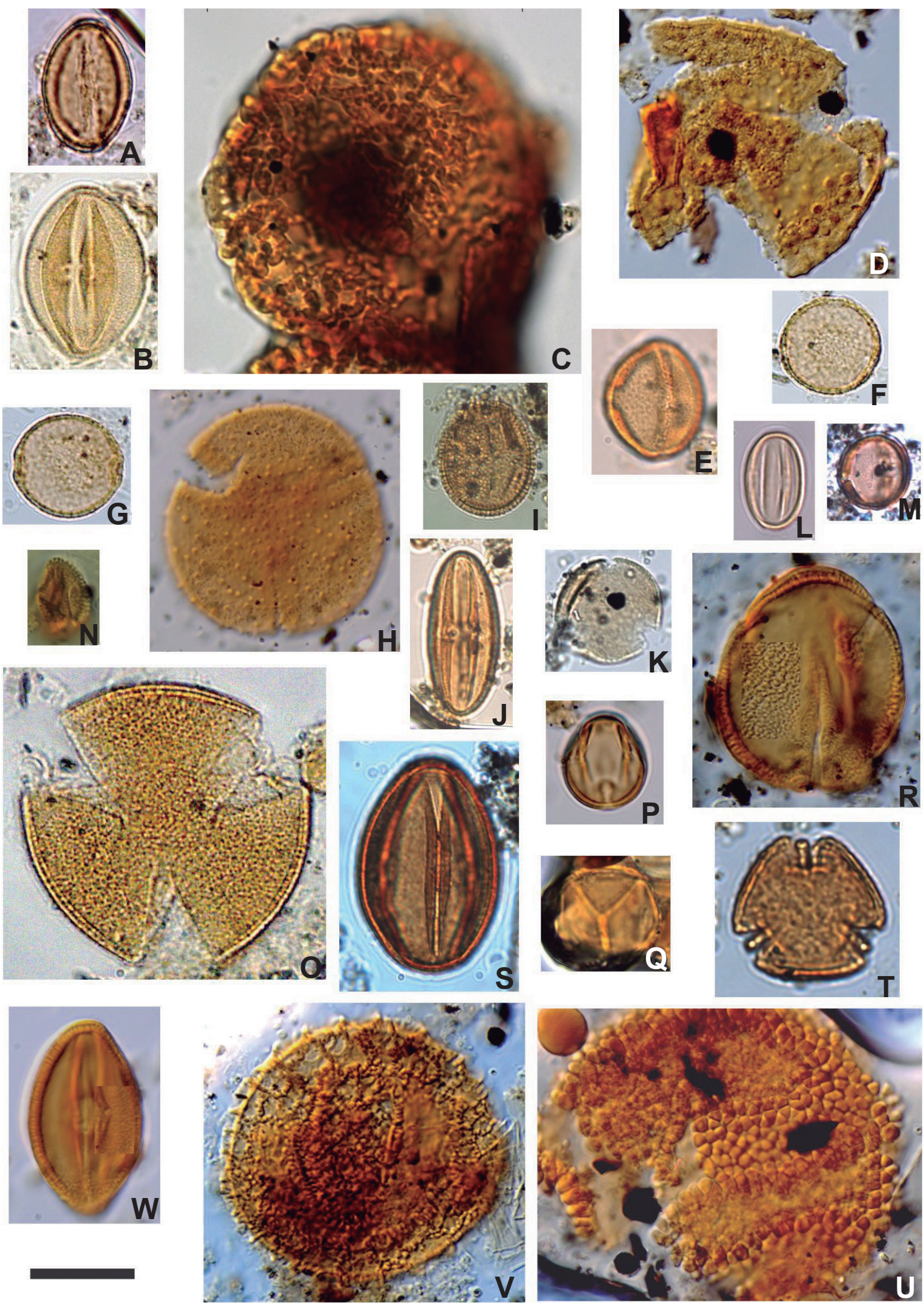

Figura 4. Fotomicrografias de grãos de pólen de angiospermas do testemunho RJ 92-5. A, Tipo/Type 36 Tabebuia; B, Tipo/Type 37 cf. Arrabidaea; C, Tipo/Type 38 Adenocalymma; D, Tipo/Type 39 Cordia; E, Tipo/Type 40 Capparis; F, Tipo/Type 41 Celtis; G, Tipo/Type 42 Trema; H, Tipo/Type 43 Tipo/Type Cactaceae; I, Tipo/Type 44 cf. Maytenus; J, Tipo/Type 45 cf. Garcinia; K, Tipo/Type 46 cf. Clusia; L, Tipo/Type 47 Combretaceae; M, Tipo/Type 48 cf. Laguncularia; N, Tipo/Type 49 Connarus; O, Tipo/Type 50 Convolvulaceae; P, Tipo/Type 51 Sloanea; Q, Tipo/Type 52 Ericaceae; R, Tipo/Type 53 Erythroxylum; S, Tipo/Type 54 Actinostemon; T, Tipo/Type 55 Alchornea; U, Tipo/Type 56 Croton; V, Tipo/Type 57 Dalechampia; W, Tipo/Type 58 Euphorbiaceae. Escala = 20 m.

Figure 4. Photomicrographs of angiosperms pollen grains of core RJ 92-5. Scale bar $=20 \mu \mathrm{m}$. 

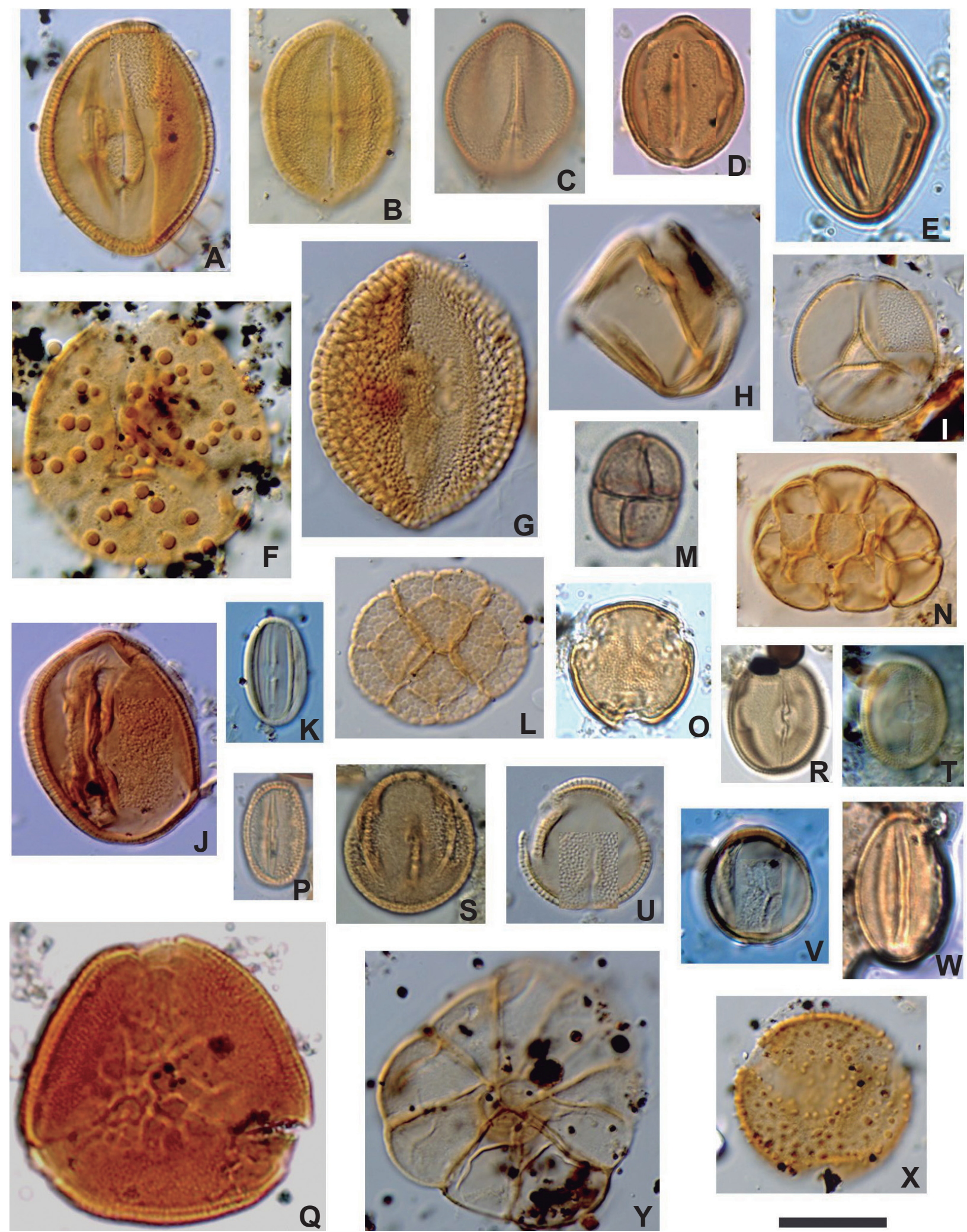

Figura 5. Fotomicrografias de grãos de pólen de angiospermas do testemunho RJ 92-5. A, Tipo/Type 59 Pachystroma; B, Tipo/Type 60 Sapium; C, Tipo/Type 61 Sebastiania; D, Tipo/Type 62 Tipo/Type Fabaceae-Caesalpinioideae 1; E, Tipo/Type 63 Fabaceae-Caesalpinioideae 2; F, Tipo/ Type 64 Bauhinia; G, Tipo/Type 65 Caesalpinia; H, Tipo/Type 66 Copaifera; I, Tipo/Type 67 cf. Melanolyxon; J, Tipo/Type 68 Peltogyne; K, Tipo/Type 69 Pterogyne; L, Tipo/Type 70 Anadenanthera; M, Tipo/Type 71 Mimosa; N, Tipo/Type 72 Piptadenia; O, Tipo/Type 73 FabaceaePapilionoideae; P. Tipo/Type 74 Acosmium; Q. Tipo/Type 75 Canavalia; R, Tipo/Type 76 Dalbergia-Machaerium; S, Tipo/Type 77 LonchocarpusOrmosia; T, Tipo/Type 78 Platymiscium; U, Tipo/Type 79 Pterocarpus; V, Tipo/Type 80 Swartzia; W, Tipo/Type 81 Zollernia; X, Tipo/Type 82 cf. Aegiphila; Y, Tipo/Type 83 Utricularia. Escala $=20 \mu \mathrm{m}$.

Figure 5. Photomicrographs of angiosperms pollen grains of core RJ 92-5. Scale bar $=20 \mu \mathrm{m}$. 

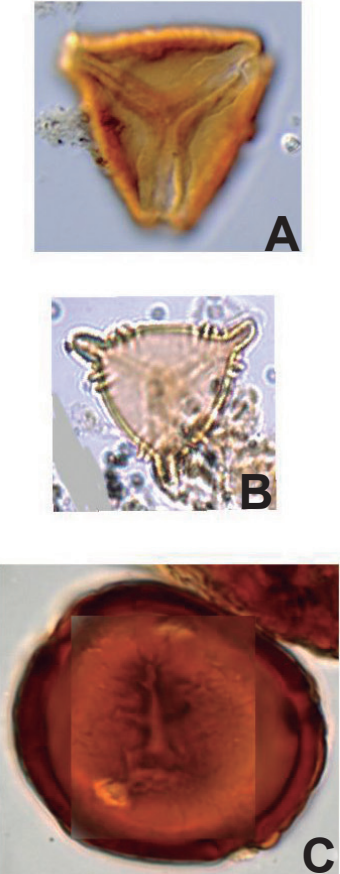

C
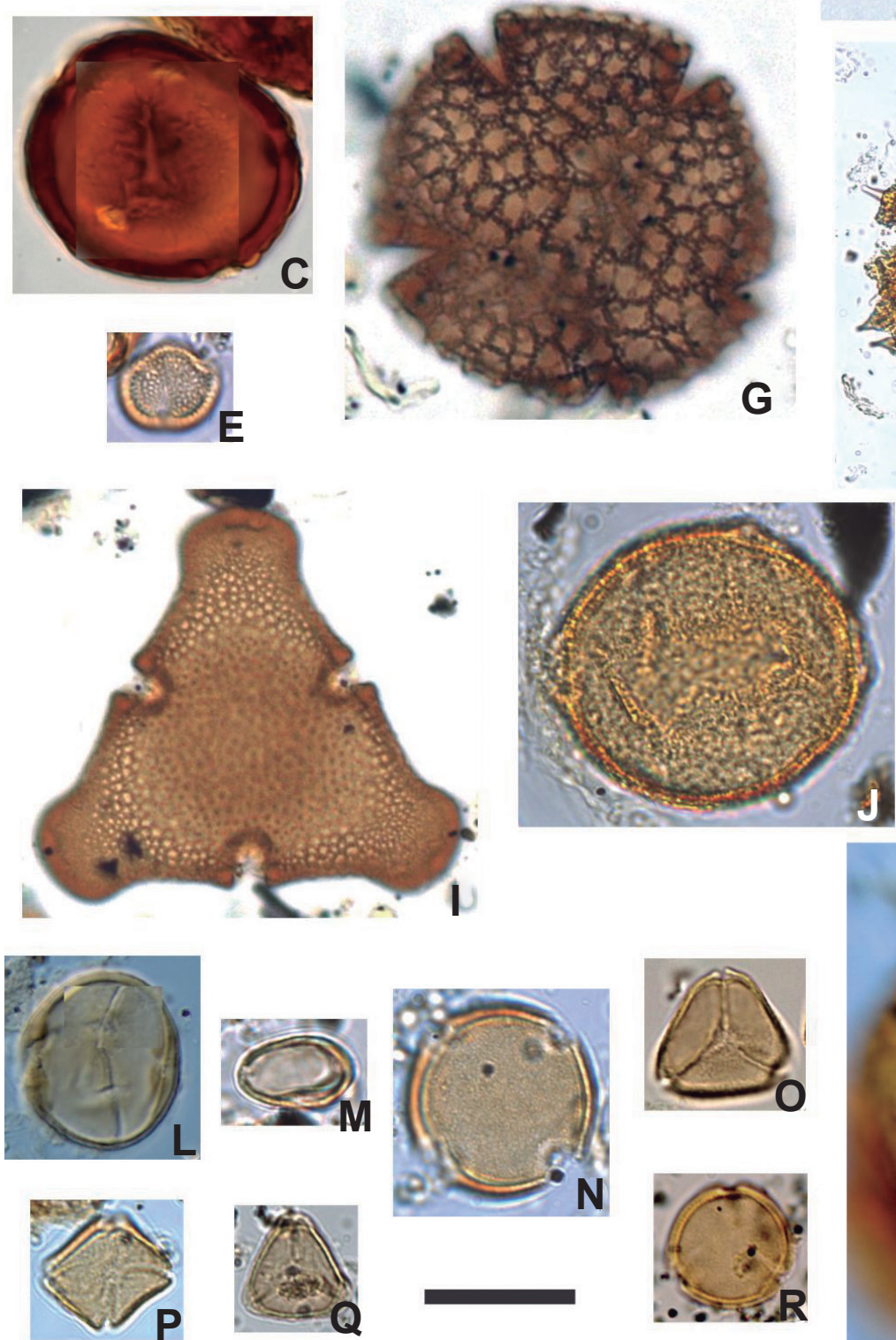

In
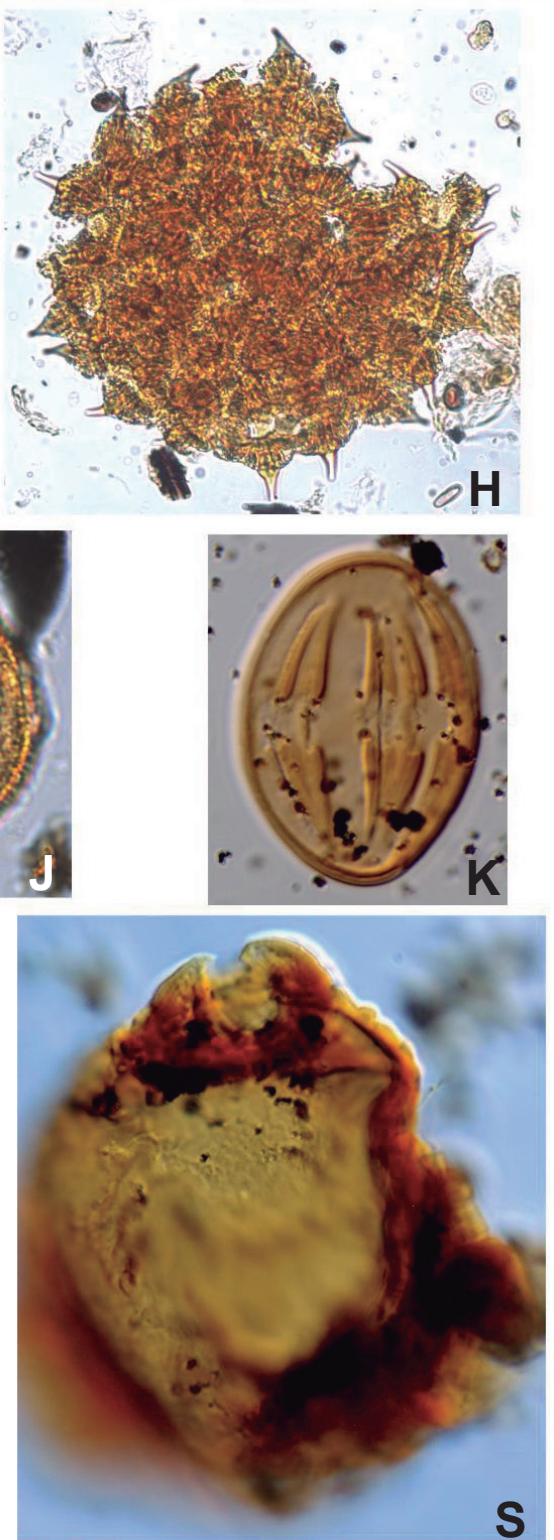

Figura 6. Fotomicrografias de grãos de pólen de angiospermas do testemunho RJ 92-5. A, Tipo/Type 84 Struthanthus; B, Tipo/Type 85 Cuphea; C, Tipo/Type 86 Malpighiaceae 1; D, Tipo/Type 87 Malpighiaceae 2; E, Tipo/Type 88 Byrsonima; F, Tipo/Type 89 Abutilon; G, Tipo/Type 90 Ceiba; H, Tipo/Type 91 Hibiscus; I, Tipo/Type 92 Pseudobombax; J, Tipo/Type 93 Waltheria; K, Tipo/Type 94 cf. Cedrela; L, Tipo/Type 95 Trichilia; M, Tipo/Type 96 cf. Ficus; N, Tipo/Type 97 Rapanea; O, Tipo/Type 98 Myrtaceae 1; P, Tipo/Type 99 Myrtaceae 2; Q, Tipo/Type 100 Psidium; R, Tipo/Type 101 Ouratea; S, Tipo/Type 102 Ludwigia. Escala $=20 \mu \mathrm{m}$.

Figure 6. Photomicrographs of angiosperms pollen grains of core RJ 92-5. Scale bar $=20 \mu \mathrm{m}$. 

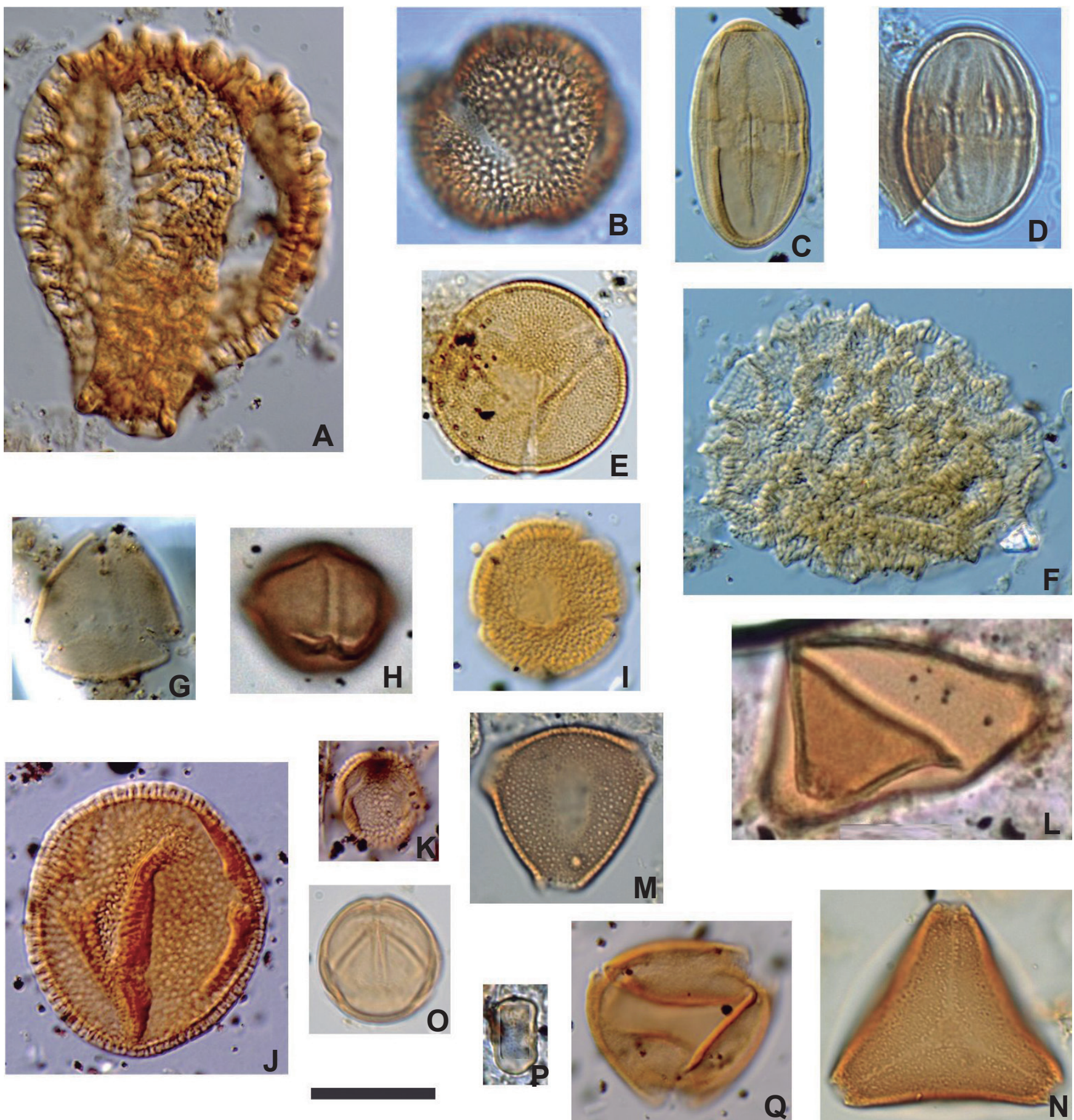

Figura 7. Fotomicrografias de grãos de pólen do testemunho RJ 92-5. A, Tipo/Type 103 Passiflora; B, Tipo/Type 104 Phyllanthaceae; C, Tipo/ Type 105 Hyeronima; D, Tipo/Type 106 Polygala; E, Tipo/Type 107 Coccoloba; F, Tipo/Type 108 Polygonum; G, Tipo/Type 109 Rhamnaceae; H, Tipo/Type 110 Rhizophora; I, Tipo/Type 111 Borreria; J, Tipo/Type 112 Psychotria; K, Tipo/Type 113 Zanthoxylum; L, Tipo/Type 114 Allophylus; M, Tipo/Type 115 Paullinia; N, Tipo/Type 116 Serjania; O, Tipo/Type 117 Solanum; P, Tipo/Type 118 cf. Cecropia; Q, Tipo/Type 119 cf. Lantana. Escala $=20 \mu \mathrm{m}$

Figure 7. Photomicrographs of angiosperms pollen grains of core RJ 92-5. Scale bar $=20 \mu \mathrm{m}$. 
Araujo, D.S.D. 1997. Cabo Frio Region. Centres of Plant Diversity: a guide and strategy for their conservation: The Americas. In: S.D.H. Davis; O. Herrera-Macbryde; J. Villa-Lobos \& A.C. Hamilton (eds.) $W W F / I U C N$, p. 373-375.

Araujo, D.S.D. 2000. Análise florística e fitogeográfica das restingas do Estado do Rio de Janeiro. Programa de Pós-Graduação em Ecologia, Universidade Federal do Rio de Janeiro, Tese de Doutorado, $176 \mathrm{p}$.

Barth, O.M. 1964. Catálogo sistemático do pólen das plantas arbóreas do Brasil meridional V: Leguminosae: Papilionatae. Memórias do Instituto Oswaldo Cruz, 62:95-125. doi:10.1590/S0074-02761964000100010

Barth, O.M. 1972a. Catálogo sistemático das plantas arbóreas do Brasil meridional XV: Myrtaceae. Memórias do Instituto Oswaldo Cruz, 70:467-496. doi:10.1590/S0074-02761972000400002

Barth, O.M. 1972b. Catálogo sistemático das plantas arbóreas do Brasil meridional XVI: Ericaceae. Memórias do Instituto Oswaldo Cruz, 70:555-569. doi:10.1590/S0074-02761972000400006

Barth, O.M. \& Barbosa,A.F. 1972a. Catálogo sistemático do pólen das plantas arbóreas do Brasil meridional XV: Myrtaceae. Memórias do Instituto Oswaldo Cruz, 70:467-496. doi:10.1590/S0074-02761972000400002

Barth, O.M. \& Barbosa, A.F. 1972b. Catálogo sistemático do pólen das plantas arbóreas do Brasil meridional XVI: Ericaceae. Memórias do Instituto Oswaldo Cruz, 70:555-569. doi:10.1590/ S0074-02761972000400006

Barth, O.M. \& Bouzada, C.P. 1964. Catálogo sistemático do pólen das plantas arbóreas do Brasil meridional VI: Leguminosae: Caesalpinioideae. Memórias do Instituto Oswaldo Cruz, 62:169192. doi:10.1590/S0074-02761964000100014

Barth, O.M.; Corte-Real, S. \& Macieira, E.G. 1976. Morfologia do pólen anemófilo e alergizante no Brasil. II. Polygonaceae, Amaranthaceae, Chenopodiaceae, Leguminosae, Euphorbiaceae e Myrtaceae. Memórias do Instituto Oswaldo Cruz, 74:191-201. doi:10.1590/S0074-02761976000300001

Barth, O.M. \& Justo, R.L. 2000. Catálogo sistemático do pólen das plantas arbóreas do Brasil meridional XXXIV: Sapotaceae. Leandra, 15:35-45.

Barth, O.M.; Justo, R.L. \& Barros, M.A. 1998. Systematic catalogue of arboreal pollen grains of southern Brazil. XXX. Meliaceae. Revista Brasileira de Biologia, 58:497-509. doi: 10.1590/S003471081998000300015

Barth, O.M. \& Melhem, T.S. 1988. Glossário Ilustrado de Palinologia. São Paulo, Editora da UNICAMP, 75 p.

Barth, O.M.; Rizzini, C.M. \& Scheel, R. 1992. Pollen morphology of Brazilian species of the genus Coccoloba P. Brown (Polygonaceae). Hoehnea, 19:65-74.

Barth, O.M. \& Silva, S.A.F. 1965. Catálogo sistemático do pólen das plantas arbóreas do Brasil meridional VII: Thymelaeaceae, Lythraceae, Lecythidaceae, Rhizophoraceae e Combretaceae. Memórias do Instituto Oswaldo Cruz, 63:255-274. doi:10.1590/ S0074-02761965000100017

Barth, O.M. \& Yoneshigue, Y. 1966. Catálogo sistemático do pólen das plantas arbóreas do Brasil meridional VIII: Leguminosae: Mimosoideae. Memórias do Instituto Oswaldo Cruz, 64:79-111. doi:10.1590/S0074-02761966000100006

Behling, H; Arz, H.W.; Pätzold, J. \& Wefer, G. 2002. Late Quaternary and Climate dynamics in Southeastern Brazil, inferences from marine slope cores GeoB 3229-2 and GeoB 3202-1. Palaeogeography, Palaeoclimatology, Palaeoecology, 179:227243. doi:10.1016/S0031-0182(01)00435-7

Carreira, L.M.M.; Silva, M.F.; Lopes, J.R.C. \& Nascimento, L.A.S. 1996. Catálogo de pólen das leguminosas da Amazônia Brasileira. Museu Paranaense Emílio Goeldi, Coleção Adolpho Ducke, 137 p.
Cordeiro, S.H. \& Lorscheitter, M.L. 1994. Palynology of lagoa dos Patos sediments, Rio Grande do Sul, Brazil. Journal of Paleolimnology, 10:35-42. doi: 10.1007/BF00683144

Costa, A.F. \& Dias, I.C.A. 2001. Flora do Parque Nacional da Restinga de Jurubatiba e arredores, Rio de Janeiro, Brasil: listagem, florística e fitogeografia. Angiospermas, pteridófitas e algas continentais. Rio de Janeiro, Museu Nacional, 200 p. (Série Livros 8).

Cruz-Barros, M.A.V., Corrêa, A.M.S. \& Makino-Watanabe, H. 2006. Estudo polínico das espécies de Aquifoliaceae, Euphorbiaceae, Lecythidaceae, Malvaceae, Phytolaccaceae e Portulacaceae ocorrentes nas restingas da Ilha do Cardoso (Cananeia, SP, Brasil). Revista Brasileira de Botânica, 29:145162. doi:10.1590/S0100-84042006000100013

Dantas, H.G.R.; Lima, H.C. \& Bohrer, C.B.A. 2009. Mapeamento da vegetação e da paisagem do município de Armação dos Búzios, Rio de Janeiro, Brasil. Rodriguésia, 60:25-38.

Erdtman, G. 1952. Pollen morphology and plant taxonomy: Angiosperms. Stockholm, Almqvist \& Wiksell, 539 p.

Erdtman, G. 1965. Pollen and spore morphology/plant taxonomy: Gymnospermae, Bryophyta. Stockholm, Almqvist \& Wiksell, 191 p.

Farág, P.R.C. 1999. Estrutura do estrato arbóreo de mata litorânea semicaducifólia sobre solo arenoso no município de Búzios, RJ. Programa de Pós-Graduação em Botânica, Museu Nacional, UFRJ, Dissertação de Mestrado, 87 p.

FEEMA. 1988. A importância da biota de Cabo Frio. Departamento de Planejamento Ambiental/ Coordenação de Dinâmica de Ecossistemas. Rio de Janeiro (datilografado), $50 \mathrm{p}$.

Fernandes, D.S. \& Sá, C.F.C. 2000. Estudo florístico preliminar das restingas remanescentes do município de Armação dos Búzios/ RJ. In: SIMPÓSIO DE ECOSSISTEMAS BRASILEIROS, 5, 2000. Anais, Vitória, v. 3, p. 57-65.

Fonseca-Kruel, V.S.; Araujo, D.S.D.; Sá, C.F.C. \& Peixoto, A.L. 2009. Quantitative ethnobotany of a restinga forest fragment in Rio de Janeiro, Brazil. Rodriguésia, 60:187-202.

Freitas, A.G. 2005. Reconstrução paleoclimática com base na análise de palinomorfos continentais em sedimentos pleistocênicos-holocênicos do talude continental brasileiro da Bacia de Campos, RJ. Programa de Pós-Graduação em Geologia, Universidade Federal do Rio de Janeiro, Dissertação de Mestrado, $126 \mathrm{p}$.

Freitas, A.G.; Carvalho, M.A. \& Mendonça Filho, J.G. 2007. Description of bryophyte and fern spores and fresh-water algae of Pleistocene-Holocene sediments of Albacora Slope, Campos Basin, RJ, Brazil. In: I.S. Carvalho; R.T. Cassab; C. Schwanke; M.A. Carvalho; A.C.S. Fernandes; M.A.C. Rodrigues; M.S.S. Carvalho; M. Arai \& M.E.Q. Oliveira (eds.) Paleontologia: Cenários de Vida, Interciência, v. 2, p. 457-470.

Gonçalves-Esteves, V. \& Cattete, D.B.D. 1997. Estudo polínico em plantas de restinga do Estado do Rio de Janeiro: Verbenaceae St. Hil. Boletim do Museu Nacional, Nota Suplementar de Botânica, 99:1-10.

Gonçalves-Esteves, V. \& Crespo, S.R.M. 1994a. Estudos polínicos em plantas de restinga do Estado do Rio de Janeiro: Bignoniaceae Juss. Boletim do Museu Nacional, Nota Suplementar de Botânica, 91:1-13.

Gonçalves-Esteves, V. \& Crespo, S.R.M. 1994b. Estudos polínicos em plantas de restinga do Estado do Rio de Janeiro: Leguminosae Juss. Boletim do Museu Nacional, Nota Suplementar de Botânica, 96:1-13.

Gonçalves-Esteves, V., Esteves, R.L. \& Oliveira, A.S. 1986. Sebastiania Spreng. (Euphorbiaceae) - Estudo polínico das 
espécies ocorrentes no Estado do Rio de Janeiro. Boletim do Museu Nacional, Nota Suplementar de Botânica, 73:1-11.

Gonçalves-Esteves, V. \& Ferreira, C.B. 1994a. Estudo polínico em plantas de restinga do Estado do Rio de Janeiro: Anacardiaceae Lindl. e Capparaceae Juss. Boletim do Museu Nacional, Nota Suplementar de Botânica, 90:1-13.

Gonçalves-Esteves, V. \& Ferreira, C.B. 1994b. Estudo polínico em plantas de restinga do Estado do Rio de Janeiro: Convolvulaceae Juss. Boletim do Museu Nacional, Nota Suplementar de Botânica, 95:1-13.

Gonçalves-Esteves, V.; Junior, E.F.S. \& Mendonça, C.B.F. 2007. Palinologia de espécies de Malpighiaceae Juss. ocorrentes nas restingas do Estado do Rio de Janeiro. Hoehnea, 34:519-529.

Gonçalves-Esteves, V.; Martins, V.L.C.; Esteves, R.L. \& Silva, S.M. 1992. Estudo polínico em plantas de restinga do Estado do Rio de Janeiro: Acanthaceae Juss. e Amaranthaceae Juss. Boletim do Museu Nacional, Nota Suplementar de Botânica, 89:1-21.

Gonçalves-Esteves, V. \& Mendonça, C.B.F. 2001. Estudo polínico em plantas de restinga do Estado do Rio de Janeiro: Clusiaceae Lindl. Revista Brasileira de Botânica, 24:527-536. doi:10.1590/ S0100-84042001000500007

Heusser, C.J. 1971. Pollen and spores of Chile - Modern types of the Pteridophyta, Gymnospermae and Angiospermae. Tucson, The University of Arizona Press, $166 \mathrm{p}$.

IBGE. 1992. Manual técnico da vegetação brasileira. Rio de Janeiro, CDDI, 92 p. (Série Manuais Técnicos de Geociências 1).

Kneller, M. \& Peteet, D. 1999. Late-Glacial to early Holocene climate changes from a central Appalachian pollen and macrofossil record. Quaternary Research, 51:133-147. doi: 10.1006/qres.1998.2026

Kurtz, B.C.; Sá, C.F.C. \& Silva, D.O. 2009. Fitossociologia do componente arbustivo-arbóreo de florestas semidecíduas costeiras da região de Emerenças, área de proteção ambiental do Pau Brasil, Armação dos Búzios, Rio de Janeiro, Brasil. Rodriguésia, 60:129-146.

Lima, H.C. 2000. Leguminosas arbóreas da Mata Atlântica. Uma análise da riqueza, padrões de distribuição geográfica e similaridades florísticas do Estado do Rio de Janeiro.

Programa de Pós-Graduação em Ecologia, Universidade Federal do Rio de Janeiro, Tese de Doutorado, 151 p.

Lobão, A.Q. \& Kurtz, B.C. 2000. Fitossociologia de um trecho de mata de restinga na Praia Gorda, município de Armação de Búzios, RJ. In: SIMPÓSIO DE ECOSSISTEMAS BRASILEIROS, 5, 2000. Anais, Vitória, v. 3, p. 66-73.

Lorenzi, H. 1998. Árvores brasileiras: manual de identificação e cultivo de plantas arbóreas nativas do Brasil. $2^{\mathrm{a}}$ ed. São Paulo, Editora Nova Odessa, v. 2, 352 p.

Lorenzi, H. 2002. Árvores brasileiras: manual de identificação e cultivo de plantas arbóreas nativas do Brasil. $4^{\mathrm{a}}$ ed. São Paulo, Editora Nova Odessa, v. 1, 368 p.

Lorenzi, H. 2009. Árvores brasileiras: manual de identificação e cultivo de plantas arbóreas nativas do Brasil. $1^{\mathrm{a}} \mathrm{ed}$. São Paulo, Editora Nova Odessa, v. 3, 384 p.

Lorscheitter, M.L. 1989. Palinologia de sedimentos quaternários do testemunho T15, Cone do Rio Grande, Atlântico Sul, Brasil. Descrições taxonômicas - parte II. Pesquisas, 22:89-127.

Lorscheitter, M.L.; Ashraf, A.R.; Windisch, P.G.; Mosbrugger, V. 1999. Pteridophytes spores of Rio Grande do Sul flora, Brazil. Part II. Paleontographica Abteilung B, 251:71-235.

Lorscheitter, M.L.; Ashraf, A.R.; Windisch, P.G. \& Mosbrugger, V. 2002. Pteridophytes spores of Rio Grande do Sul flora, Brazil. Part IV. Paleontographica Abteilung B, 263:1-159.
Lorscheitter, M.L.; Ashraf, A.R.; Windisch, P.G. \& Mosbrugger, V. 2005. Pteridophytes spores of Rio Grande do Sul flora, Brazil. Part V. Paleontographica Abteilung B, 270:1-180.

Luz, C.F.P. \& Barth, O.M. 2000. Palinomorfos indicadores de tipos de vegetação em sedimentos holocênicos da Lagoa de Cima, Norte do Estado do Rio de Janeiro, Brasil - Dicotyledoneae. Leandra, 15:11-34.

Luz, C.F.P. \& Barth, O.M. 2002. Palinomorfos indicadores de tipos de vegetação em sedimentos holocênicos da Lagoa de Cima, Norte do Estado do Rio de Janeiro, Brasil - Monocotyledoneae, Lycopodophyta, Pteridophyta e Bryophyta. Leandra, 17:7-22.

Luz, C.F.P.; Barth, O.M.; Martin, L.; Silva, C.G. \& Turcq, J. 2011. Palynological evidence of the replacement of the hygrophilous forest by field vegetation during the last 7,000 years B.P. in the northern coast of Rio de Janeiro, Brazil. Anais da Academia Brasileira de Ciências, 83:939-951. doi:10.1590/S000137652011000300001

Luz, C.F.P.; Barth, O.M. \& Silva, C.G. 2006. Dinâmica temporal na lagoa do Campelo, região norte do Estado do Rio de Janeiro, baseada em estudos palinológicos. Revista Brasileira de Paleontologia, 9:127-136. doi:10.4072/rbp.2006.1.13

Martin, L.; Tasayco-Ortega, L.A.; Flexor, J.M.; Suguio, K. \& Turcq, B. 1996. Registro das variações do nível do mar e do clima nos sedimentos das pequenas lagunas hipersalinas de Cabo Frio (RJ). In: CONGRESSO BRASILEIRO DE GEOLOGIA, 39, 1996. Anais, Sergipe-Bahia, SBG, p. 523-525.

Medeanic, S.; Costa César, S.B. \& Corrêa, I.C.S. 2006. Os palinomorfos nas reconstruções paleoambientais: Azolla filiculoides Lam. nos sedimentos holocênicos da planície costeira do Rio Grande do Sul. Gravel, 4:49-61.

Mendonça, C.B.F. \& Gonçalves-Esteves, V. 2000a. Palinologia de espécies da Tribo Eupatorieae (Compositae Giseke) ocorrentes na Restinga de Carapebus, Rio de Janeiro. Revista Brasileira de Botânica, 23:195-205. doi:10.1590/S0100-84042000000200009

Mendonça, C.B.F. \& Gonçalves-Esteves, V. 2000b. Morfologia polínica de algumas espécies da Tribo Vernonieae (Compositae Giseke) da Restinga de Carapebus, Rio de Janeiro. Hoehnea, 27:131-142.

Milward-de-Azevedo, M.A.; Gonçalves-Esteves, V. \& Baumgratz, J.F. 2004. Palinotaxonomia das espécies de Passiflora L. subg. Dacaloba (DC.) Rchb. (Passifloraceae) no sudeste do Brasil. Revista Brasileira de Botânica, 27:655-665. doi:10.1590/S010084042004000400006

Moreira, F.F.; Mendonça, C.B.F.; Pereira, J.F. \& Gonçalves-Esteves, V. 2004. Palinotaxonomia de espécies de Apocynaceae ocorrentes na Restinga de Carapebus, Carapebus, RJ. Acta Botanica Brasilica, 18:711-721. doi:10.1590/S0102-33062004000400002

Muller, J. \& Caratini, C. 1977. Pollen of Rhizophora (Rhizophoraceae) as a guide fossil. Pollen et Spores, 19:361-389.

Neves, P.C.P. \& Lorscheitter, M.L. 1991. Upper Quaternary palaeoenvironments in the northern coastal plain of Rio Grande do Sul, Brazil. Quaternary of South America and Antartic Peninsula, 9:39-67.

Projeto Radambrasil. 1983. Levantamento de Recursos Naturais. Folhas Rio de Janeiro/Vitória (SF.23 / 24).

Punt, W.; Hoen, P.P.; Blackmore, S.; Nilsson, S. \& Thomas, A. 2007. Glossary of pollen and spore terminology. Review of Palaeobotany and Palynology, 143:1-81. doi:10.1016/j. revpalbo.2006.06.008

Reimer, P.J.; Baillie, M.G.L.; Bard, E.; Bayliss, A.; Beck, J.W.; Bertrand, C.; Blackwell, P.G. \& Buck, C.E. 2004. IntCal04 terrestrial radiocarbon age calibration, 0-26 Cal Kyr BP. 
Radiocarbon, 46:1029-1058.

Roubik, D.W. \& Moreno, J.E. 1991. Pollen and spores of Barro Colorado Island. Missouri Botanical Garden, 36:1-270 (Monographs in Systematic Botany).

Sá, C.F.C. 2002. Regeneração de um trecho de floresta de restinga na Reserva Ecológica Estadual de Jacarepiá, Saquarema, Estado do Rio de Janeiro: II - Estrato arbustivo. Rodriguésia, 53:5-23.

Sá, C.F.C. 2006. Estrutura, diversidade e conservação de angiospermas no Centro de Diversidade de Cabo Frio, Rio de Janeiro. Programa de Programa de Pós-Graduação em Ecologia, Universidade Federal do Rio de Janeiro, Tese de Doutorado, 251p.

Salgado-Labouriau, M.L. 1973. Contribuição à Palinologia dos Cerrados. Editora da Academia Brasileira de Ciências, Rio de Janeiro, $291 \mathrm{p}$.

Scheel, R.; Ybert, J-P. \& Barth, O.M. 1996. Pollen morphology of the Boraginaceae from Santa Catarina State (southern Brazil), with comments on the taxonomy of the family. Grana, 35:138153. doi:10.1080/00173139609429075

Souza, F.C.; Mendonça, C.B.F. \& Gonçalves-Esteves, V. 2004a. Estudo polínico de espécies de Pitcairnioideae e Tilandsioideaeae (Bromeliaceae Juss.) ocorrentes na Restinga de Carapebus, Estado do Rio de Janeiro. Arquivos do Museu Nacional, 62:15-23.

Souza, F.C.; Souza, M.A.; Mendonça, C.B.F. \& Gonçalves-Esteves, V. 2004b. Estudo polínico de espécies de Aeschynomeneae e Phasedeae (Papilionoideae - Leguminosae Juss.) ocorrentes nas restingas do Estado do Rio de Janeiro. Arquivos do Museu Nacional, 62:357-366.

Souza, V.C. \& Lorenzi, H. 2008. Botânica sistemática: guia ilustrado para identificação das famílias de fanerógamas nativas $e$ exóticas no Brasil, baseado na APG II. $2^{\mathrm{a}}$ ed. São Paulo, Editora Nova Odessa, 703 p.

Stuiver, M. \& Reimer, P.J. 1993. Extended ${ }^{14} \mathrm{C}$ data base and revised CALIB 3.0 ${ }^{14} \mathrm{C}$ age calibration program. Radiocarbon, 35:215-230.

Tasayaco-Ortega, L. 1996. Variations paléohidrologiques et paléoclimatiques d'une région d'upwelling au cours de l'Holocene: enregistrement dans les lagunes côtière de Cabo Frio (État de Rio de Janeiro, Brésil). Université Paris VI, Thèse de Doctorato, 322p.

Toledo, M.B.; Barth, O.M.; Silva, C.G. \& Barros, M.A. 2009. Testing multivariate analysis in paleoenvironmental reconstructions using pollen records from lagoa Salgada, NE, Rio de Janeiro State, Brazil. Anais da Academia Brasileira de Ciências, 81:757-768.

Ururahy, J.C.C.; Collares, J.E.R. \& Santos, M.M. 1987. Nota sobre a formação fisionômico-ecológica disjunta da estepe nordestina na área do pontal de Cabo Frio, RJ. Revista Brasileira de Geografia, 49:25-29.

Willard, D.A.; Benhardt, C.E.; Weimer, L.; Cooper, S.R., C.M. \& Gamez, D. 2004. Atlas of pollen and spores of the Florida Everglades. Palynology, 28:175-227. doi:10.1080/01916122.2 004.9989597

Ybert, J.P. 1979. Atlas de pollens de Côte D'Ivoire. Paris, Orstom, $40 \mathrm{p}$.

Ybert, J.P.; Salgado-Labouriau, M.L.; Barth, O.M.; Lorscheitter, M.L.; Barros, M.A.; Chaves, S.A.M.; Luz, C.F.P.; Ribeiro, M.; Scheel, R. \& Vicentini, K.R.F. 1992. Sugestão para padronização da metodologia empregada para estudos palinológicos do Quaternário. Revista do Instituto Geológico da USP, 13:47-49.

Received in July, 2011; accepted in October, 2012.

Apêndice 1. Descrições morfológicas dos grãos de pólen e esporos identificados no testemunho RJ 92-5, lagoa da Ferradura, Armação dos Búzios, RJ. Abreviaturas: Cl, colpo; D, diâmetro; E, eixo equatorial; EM, eixo equatorial maior; Em, eixo equatorial menor; Enc, comprimento da endoabertura; Enl, largura da endoabertura; Epa, altura do espinho; Epl, largura do espinho; Ex, espessura da exina; P, eixo polar; Pe, perina; $\operatorname{Pr}$, poro.

Appendix 1. Morphological descriptions of pollen grains and spores identified in core RJ 92-5, Ferradura lagoon, Armação dos Búzios, RJ. Abbreviations: Cl, colpus; D, diameter; E, equatorial axis; EM, major equatorial axis; Em, minor equatorial axis; Enc, endoaperture length; Enl, endoaperture width; Epa, espine height; Epl, espine width; Ex, exine; $\mathbf{P}$, polar axis; Pe, perine; Pr, pore.

\begin{tabular}{|c|c|c|c|c|c|c|c|}
\hline Tipos polínicos & $\begin{array}{l}\text { Unidade } \\
\text { polínica }\end{array}$ & $\begin{array}{l}\text { Polaridade/ } \\
\text { Simetria }\end{array}$ & Medidas $(\mu \mathrm{m})$ & $\begin{array}{c}\text { NPC } \\
\text { aberturas/ } \\
\text { Cicatriz }\end{array}$ & Exina/ Perina & $\begin{array}{l}\text { Forma/ } \\
\text { Âmbito }\end{array}$ & Observações \\
\hline \multicolumn{8}{|l|}{ Briófitas } \\
\hline \multicolumn{8}{|l|}{ Anthocerotageae } \\
\hline $\begin{array}{l}\text { Tipo } 1 \text { Phaeoceros } \\
\text { L. Prosk (Figura 2A) }\end{array}$ & mônade & radiossimétrico & $E=45 \times 45$ & trilete & $\begin{array}{l}\text { equinada/ } \\
\text { ausente }\end{array}$ & $\begin{array}{l}\text { circular a } \\
\text { subtriangular }\end{array}$ & $\begin{array}{c}\text { bifurcação nas } \\
\text { extremidades dos raios }\end{array}$ \\
\hline \multicolumn{8}{|l|}{ Sphagnaceae } \\
\hline $\begin{array}{l}\text { Tipo } 2 \text { Sphagnum } \\
\text { [Dill.] Hedwing (Figura 2B) }\end{array}$ & mônade & radiossimétrico & $E=40 \times 40$ & trilete & $\begin{array}{l}\text { psilada/ } \\
\text { ausente }\end{array}$ & $\begin{array}{l}\text { triangular/ } \\
\text { equatorial }\end{array}$ & \\
\hline \multicolumn{8}{|l|}{ Pteridófitas } \\
\hline \multicolumn{8}{|l|}{ Azollaceae } \\
\hline $\begin{array}{l}\text { Tipo } 3 \text { Azolla } \\
\text { Lam., } 1783 \text { (Figura 2C) }\end{array}$ & & & $E=54 \times 49$ & & $\begin{array}{l}\text { granulada/ } \\
\text { ausente }\end{array}$ & $\begin{array}{l}\text { irregular a } \\
\text { subcircular }\end{array}$ & $\begin{array}{l}\text { mássula com gloquídios } \\
\text { com extrremidade apical } \\
\text { em forma de âncoras }\end{array}$ \\
\hline \multicolumn{8}{|l|}{ Aspleniaceae } \\
\hline $\begin{array}{l}\text { Tipo } 4 \text { Asplenium } \\
\text { L., } 1753 \text { (Figura 2D) }\end{array}$ & mônade & $\begin{array}{l}\text { bilateral/ plano- } \\
\text { convexo }\end{array}$ & $\begin{array}{c}\mathrm{P}=44 \times \mathrm{EM}=35 \\
\mathrm{Ex}=2 ; \mathrm{Pe}=4.5\end{array}$ & monolete & $\begin{array}{l}\text { psilada/ } \\
\text { reticulada }\end{array}$ & equatorial & \\
\hline \multicolumn{8}{|l|}{ Blechnaceae } \\
\hline $\begin{array}{l}\text { Tipo } 5 \text { Blechnum } \\
\text { L., } 1753 \text { (Figura 2E) }\end{array}$ & mônade & $\begin{array}{c}\text { bilateral/ plano- } \\
\text { convexo }\end{array}$ & $\begin{array}{c}\mathrm{P}=30 \times \mathrm{Em}=22 \\
\mathrm{Ex}=2\end{array}$ & monolete & $\begin{array}{l}\text { psilada/ } \\
\text { granulada }\end{array}$ & equatorial & \\
\hline
\end{tabular}


Apêndice 1. Continua.

\begin{tabular}{|c|c|c|c|c|c|c|c|}
\hline Tipos polínicos & $\begin{array}{l}\text { Unidade } \\
\text { polínica }\end{array}$ & $\begin{array}{l}\text { Polaridade/ } \\
\text { Simetria }\end{array}$ & Medidas $(\mu \mathrm{m})$ & $\begin{array}{c}\text { NPC } \\
\text { aberturas/ } \\
\text { Cicatriz }\end{array}$ & Exina/ Perina & $\begin{array}{l}\text { Forma/ } \\
\text { Âmbito }\end{array}$ & Observações \\
\hline \multicolumn{8}{|l|}{ Cyatheaceae } \\
\hline $\begin{array}{l}\text { Tipo } 6 \text { Cyathea } \\
\text { Sm., } 1793 \text { (Figura 2F) }\end{array}$ & mônade & radiossimétrico & $\begin{array}{c}\mathrm{P}=60 \times \mathrm{E}=60 \\
\mathrm{Ex}=2.5\end{array}$ & trilete & $\begin{array}{l}\text { perfurada/ } \\
\text { ausente }\end{array}$ & triangular & \\
\hline \multicolumn{8}{|l|}{ Polypodiaceae } \\
\hline $\begin{array}{l}\text { Tipo } 7 \text { Polypodium } 1 \\
\text { (Figura } 2 \mathrm{G} \text { ) }\end{array}$ & mônade & $\begin{array}{l}\text { bilateral/ plano- } \\
\text { convexo }\end{array}$ & $\begin{array}{c}\mathrm{EM}=70 \times \mathrm{P}=42 \\
\mathrm{Ex}=4\end{array}$ & monolete & $\begin{array}{l}\text { verrucada/ } \\
\text { ausente }\end{array}$ & equatorial & \\
\hline $\begin{array}{l}\text { Tipo } 8 \text { Polypodium } 2 \\
\text { (Figura } 2 \mathrm{H} \text { ) }\end{array}$ & mônade & radiossimétrico & $\begin{array}{c}\mathrm{P}=45 \times \mathrm{E}=42 \\
\quad \mathrm{Ex}=3.5\end{array}$ & trilete & $\begin{array}{l}\text { verrucada/ } \\
\text { ausente }\end{array}$ & triangular & \\
\hline \multicolumn{8}{|l|}{ Pteridaceae } \\
\hline $\begin{array}{l}\text { Tipo } 9 \text { Acrostichum } \\
\text { L., } 1753 \text { (Figura 2I) }\end{array}$ & mônade & radiossimétrico & $P=73 \times E=60 ; E x=2$ & trilete & $\begin{array}{l}\text { psilada/ } \\
\text { granulada }\end{array}$ & $\begin{array}{l}\text { subtriangular/ } \\
\text { equatorial }\end{array}$ & \\
\hline $\begin{array}{l}\text { Tipo } 10 \text { Pityrogramma } \\
\text { Link, } 1833 \text { (Figura 2J) }\end{array}$ & mônade & radiossimétrico & $\begin{array}{c}\mathrm{P}=40 \times \mathrm{E}=40 \\
\mathrm{Ex}=3.5\end{array}$ & trilete & $\begin{array}{l}\text { rugulada e } \\
\text { verrucada }\end{array}$ & $\begin{array}{l}\text { subtriangular/ } \\
\text { equatorial }\end{array}$ & \\
\hline \multicolumn{8}{|l|}{ Gimnospermas } \\
\hline \multicolumn{8}{|l|}{ Podocarpaceae } \\
\hline $\begin{array}{l}\text { Tipo } 11 \text { Podocarpus } \\
\text { L'Hér. ex Pers., } 1807 \text { (Figura } \\
\text { 3A) }\end{array}$ & mônade & bilateral & $\begin{array}{c}\mathrm{P}=56 \times \mathrm{E}=38 \text { (total); } \\
\mathrm{P}=23 \times \mathrm{XE}=20 \text { (corpo); } \\
\mathrm{P}=33 \times \mathrm{E}=18(\text { sacos })\end{array}$ & & reticulada & elíptica & \\
\hline \multicolumn{8}{|l|}{$\begin{array}{l}\text { Angiospermas - } \\
\text { Monocotiledôneas }\end{array}$} \\
\hline \multicolumn{8}{|l|}{ Alismataceae } \\
\hline $\begin{array}{l}\text { Tipo } 12 \text { Sagittaria } \\
\text { Rupp. ex L., } 1753 \text { (Figura 3B) }\end{array}$ & mônade & $\begin{array}{c}\text { apolar- } \\
\text { assimétrico }\end{array}$ & $\mathrm{D}=25 \times 25$ & 5-porado & equinada & circular & \\
\hline \multicolumn{8}{|l|}{ Arecaceae } \\
\hline $\begin{array}{l}\text { Tipo } 13 \text { Allagoptera } \\
\text { Nees, } 1821 \text { (Figura 3C) }\end{array}$ & mônade & $\begin{array}{l}\text { heteropolar- } \\
\text { bilateral }\end{array}$ & $\begin{array}{l}\mathrm{P}=53 \times \mathrm{E}=30 \\
\mathrm{Cl}=48 ; \mathrm{Ex}=2\end{array}$ & monocolpado & escabrada & prolato & \\
\hline \multicolumn{8}{|l|}{ Bromeliaceae } \\
\hline $\begin{array}{l}\text { Tipo } 14 \text { Bromeliaceae } \\
\text { (Figura 3D) }\end{array}$ & mônade & $\begin{array}{l}\text { heteropolar- } \\
\text { elíptico }\end{array}$ & $\begin{array}{l}\mathrm{P}=43 \times \mathrm{E}=29 \\
\mathrm{Cl}=33 ; \mathrm{Ex}=1\end{array}$ & monocolpado & $\begin{array}{l}\text { reticulada- } \\
\text { homobrocada }\end{array}$ & equatorial & colpo longo \\
\hline \multicolumn{8}{|l|}{ Cyperaceae } \\
\hline $\begin{array}{l}\text { Tipo } 15 \text { cf. Bulbostylis } \\
\text { D C., } 1836 \text { (Figura 3E) }\end{array}$ & mônade & $\begin{array}{l}\text { heteropolar- } \\
\text { assimétrico }\end{array}$ & $P=40 ; E=27 ; E x=2$ & $\begin{array}{l}5 \text { zonas } \\
\text { aperturais } \\
\text { (poroides) }\end{array}$ & escabrada & cônico & \\
\hline \multicolumn{8}{|l|}{ Poaceae } \\
\hline $\begin{array}{l}\text { Tipo } 16 \text { Poaceae } \\
\text { (Figura 3F) }\end{array}$ & mônade & $\begin{array}{l}\text { heteropolar- } \\
\text { radiossimétrico }\end{array}$ & $\begin{array}{c}\mathrm{P}=59 ; \mathrm{E}=46 ; \operatorname{Pr}=9 ; \\
\mathrm{Ex}=2\end{array}$ & monoporado & $\begin{array}{l}\text { psilada e } \\
\text { escabrada }\end{array}$ & circular & poro com ânulo conspícuo \\
\hline \multicolumn{8}{|l|}{ Ruppiaceae } \\
\hline $\begin{array}{l}\text { Tipo } 17 \text { Ruppia } \\
\text { L., } 1753 \text { (Figura 3G) }\end{array}$ & mônade & $\begin{array}{l}\text { heteropolar- } \\
\text { bilateral }\end{array}$ & $\mathrm{EM}=250 ; \mathrm{Em}=120$ & inaperturado & $\begin{array}{c}\text { reticulada- } \\
\text { heterobrocada }\end{array}$ & subcilíndrico & \\
\hline \multicolumn{8}{|l|}{ Typhaceae } \\
\hline $\begin{array}{l}\text { Tipo } 18 \text { Typha } \\
\text { L., } 1753 \text { (Figura 3H) }\end{array}$ & mônade & $\begin{array}{l}\text { heteropolar- } \\
\text { radiossimétrico }\end{array}$ & $\mathrm{EM}=30 \times \mathrm{Em}=26$ & monoporado & reticulada & circular & \\
\hline \multicolumn{8}{|l|}{$\begin{array}{l}\text { Angiospermas - } \\
\text { Eudicotiledôneas } \\
\text { Core Eudicotiledôneas }\end{array}$} \\
\hline \multicolumn{8}{|l|}{ Acanthaceae } \\
\hline $\begin{array}{l}\text { Tipo } 19 \text { Avicennia } \\
\text { L., } 1753 \text { (Figura 3I) }\end{array}$ & mônade & $\begin{array}{l}\text { isopolar- } \\
\text { radiossimétrico }\end{array}$ & $P=17 \times E=16 ; E x=1$ & tricolporado & microrreticulada & $\begin{array}{l}\text { prolato } \\
\text { esferoidal }\end{array}$ & poros circulares \\
\hline $\begin{array}{l}\text { Tipo } 20 \text { Justicia } \\
\text { L., } 1753 \text { (Figura 3J) }\end{array}$ & mônade & $\begin{array}{l}\text { isopolar- } \\
\text { radiossimétrico }\end{array}$ & $\begin{array}{c}\mathrm{P}=56 \times \mathrm{E}=30 \\
\operatorname{Pr}=5.5 \times 3.5 ; \mathrm{Ex}=3\end{array}$ & $\begin{array}{l}\text { tricolporado- } \\
\text { heterocolpado }\end{array}$ & reticulada & prolato & \\
\hline \multicolumn{8}{|l|}{ Amaranthaceae } \\
\hline $\begin{array}{l}\text { Tipo } 21 \text { Alternanthera } \\
\text { Forssk., } 1775 \text { (Figura } 3 \mathrm{~K} \text { ) }\end{array}$ & mônade & $\begin{array}{l}\text { apolar- } \\
\text { radiossimétrico }\end{array}$ & $\begin{array}{c}\mathrm{P}=18 \times \mathrm{E}=19 \\
\quad \mathrm{Ex}=1.5\end{array}$ & periporado & $\begin{array}{l}\text { lofada com } \\
\text { báculos }\end{array}$ & esferoidal & $\begin{array}{l}8 \text { a } 12 \text { malhas hexagonais } \\
\text { grandes por face }\end{array}$ \\
\hline $\begin{array}{l}\text { Tipo } 22 \text { Gomphrena } \\
\text { L., } 1753 \text { (Figura 3L) }\end{array}$ & mônade & $\begin{array}{l}\text { apolar- } \\
\text { radiossimétrico }\end{array}$ & $\begin{array}{c}\mathrm{EM}=31 \times \mathrm{Em}=29 \\
\mathrm{Ex}=4.5\end{array}$ & periporado & $\begin{array}{l}\text { reticulo- } \\
\text { baculada }\end{array}$ & esferoidal & 12 a 20 malhas grandes \\
\hline $\begin{array}{l}\text { Tipo } 23 \text { Chenopodium } \\
\text { L., } 1753 \text { (Figura 3M) }\end{array}$ & mônade & $\begin{array}{l}\text { apolar- } \\
\text { radiossimétrico }\end{array}$ & $\begin{array}{c}\mathrm{EM}=32 ; \mathrm{Em}=30.5 \\
\mathrm{Pr}=2 \times 2 ; \mathrm{Ex}=1.5\end{array}$ & periporado & escabrada & esferoidal & $>35$ poros por face \\
\hline \multicolumn{8}{|l|}{ Anacardiaceae } \\
\hline $\begin{array}{l}\text { Tipo } 24 \text { Anacardiaceae } \\
\text { (Figura } 3 \mathrm{~N} \text { ) }\end{array}$ & mônade & $\begin{array}{l}\text { isopolar- } \\
\text { radiossimétrico }\end{array}$ & $\begin{array}{c}\mathrm{P}=35 \times \mathrm{E}=25 \\
\mathrm{P} / \mathrm{E}=1,40 ; \mathrm{Ex}=1\end{array}$ & tricolporado & $\begin{array}{l}\text { estriado- } \\
\text { reticulada }\end{array}$ & prolato & poros lalongados \\
\hline
\end{tabular}


Apêndice 1. Continua.

\begin{tabular}{|c|c|c|c|c|c|c|c|}
\hline Tipos polínicos & $\begin{array}{l}\text { Unidade } \\
\text { polínica }\end{array}$ & $\begin{array}{l}\text { Polaridade/ } \\
\text { Simetria }\end{array}$ & Medidas $(\mu \mathrm{m})$ & $\begin{array}{c}\text { NPC } \\
\text { aberturas/ } \\
\text { Cicatriz }\end{array}$ & Exina/ Perina & $\begin{array}{l}\text { Forma/ } \\
\text { Âmbito }\end{array}$ & Observações \\
\hline \multicolumn{8}{|l|}{ Anacardiaceae } \\
\hline $\begin{array}{l}\text { Tipo } 25 \text { Astronium } \\
\text { Jacq., } 1760 \text { (Figura 30) }\end{array}$ & mônade & $\begin{array}{l}\text { isopolar- } \\
\text { radiossimétrico }\end{array}$ & $\begin{array}{c}\mathrm{P}=36 \times \mathrm{E}=22 \\
\mathrm{P} / \mathrm{E}=1,63 ; \mathrm{Ex}=2\end{array}$ & tricolporado & $\begin{array}{l}\text { estriado- } \\
\text { reticulada, } \\
\text { heterobrocada }\end{array}$ & prolato & poros lalongados \\
\hline $\begin{array}{l}\text { Tipo } 26 \text { Schinus } \\
\text { L., } 1753 \text { (Figura 3P) }\end{array}$ & mônade & $\begin{array}{l}\text { isopolar- } \\
\text { radiossimétrico }\end{array}$ & $\begin{array}{c}P=30 \times E=20 \\
P / E=1,50 ; E x=1\end{array}$ & tricolporado & $\begin{array}{c}\text { estriado- } \\
\text { reticulada, } \\
\text { heterobrocada }\end{array}$ & prolato & poros lalongados \\
\hline $\begin{array}{l}\text { Tipo } 27 \text { Tapirira } \\
\text { Aubl., } 1775 \text { (Figura 3Q) }\end{array}$ & mônade & $\begin{array}{l}\text { isopolar- } \\
\text { radiossimétrico }\end{array}$ & $\begin{array}{c}P=25 \times E=16 \\
P / E=1,56 ; E x=2\end{array}$ & tricolporado & estriada & prolato & $\begin{array}{l}\text { poros lalongados; estrias } \\
\text { perpendiculares }\end{array}$ \\
\hline $\begin{array}{l}\text { Tipo } 28 \text { Spondias } \\
\text { L., } 1753 \text { (Figura 3R) }\end{array}$ & mônade & $\begin{array}{l}\text { isopolar- } \\
\text { radiossimétrico }\end{array}$ & $\begin{array}{c}P=46 \times E=25 \\
P / E=1,84 ; E x=2\end{array}$ & tricolporado & estriada & prolato & poros lalongados \\
\hline \multicolumn{8}{|l|}{ Apocynaceae } \\
\hline $\begin{array}{l}\text { Tipo } 29 \text { Apocynaceae } \\
\text { (Figura 3S) }\end{array}$ & mônade & $\begin{array}{l}\text { isopolar- } \\
\text { radiossimétrico }\end{array}$ & $\begin{array}{c}\mathrm{P}=31 \times \mathrm{E}=26 ; \mathrm{P} / \mathrm{E}= \\
0,78 ; \mathrm{Pr}=4 \times 2 ; \mathrm{Ex}=1.5\end{array}$ & trizonoporado & escabrada & suboblato & \\
\hline \multicolumn{8}{|l|}{ Aquifoliaceae } \\
\hline $\begin{array}{l}\text { Tipo } 30 \text { Ilex } \\
\text { L., } 1753 \text { (Figura 3T) }\end{array}$ & mônade & $\begin{array}{l}\text { isopolar- } \\
\text { radiossimétrico }\end{array}$ & $\begin{array}{c}\mathrm{P}=22 \times \mathrm{E}=18 \\
\mathrm{P} / \mathrm{E}=1.22\end{array}$ & tricolporado & clavada-pilada & subprolato & cólporos lalongados \\
\hline \multicolumn{8}{|l|}{ Araliaceae } \\
\hline $\begin{array}{l}\text { Tipo } 31 \text { Dendropanax } \\
\text { Decne. \& Planch,1854 (Figura 3U) }\end{array}$ & mônade & $\begin{array}{l}\text { isopolar- } \\
\text { radiossimétrico }\end{array}$ & $\begin{aligned} \mathrm{P} & =35 \times \mathrm{E}=28 \\
\mathrm{P} / \mathrm{E} & =1.25 ; \mathrm{Ex}=1.5\end{aligned}$ & tricolporado & granulada & subprolato & \\
\hline $\begin{array}{l}\text { Tipo } 32 \text { Hydrocotyle } \\
\text { L., } 1753 \text { (Figura 3V) }\end{array}$ & mônade & $\begin{array}{l}\text { isopolar- } \\
\text { radiossimétrico }\end{array}$ & $\begin{aligned} \mathrm{P} & =26 \times \mathrm{E}=14 \\
\mathrm{P} / \mathrm{E} & =1.85 ; \mathrm{Ex}=1.5\end{aligned}$ & tricolporado & escabrada & prolato & \\
\hline \multicolumn{8}{|l|}{ Asteraceae } \\
\hline $\begin{array}{l}\text { Tipo } 33 \text { Tribo Vernonieae } \\
\text { (Figura } 3 \mathrm{~W} \text { ) }\end{array}$ & mônade & $\begin{array}{l}\text { isopolar- } \\
\text { radiossimétrico }\end{array}$ & $\begin{array}{c}\mathrm{P}=36 \times \mathrm{E}=32 ; \\
\mathrm{P} / \mathrm{E}=1.12 ; \mathrm{Ex}=6.5 \\
\mathrm{Epa}=5 ; \mathrm{Epl}=2\end{array}$ & tricolporado & equinolofada & $\begin{array}{l}\text { prolato } \\
\text { esferoidal }\end{array}$ & \\
\hline $\begin{array}{l}\text { Tipo } 34 \text { Tribo Eupatorieae } \\
\text { (Figura } 3 \mathrm{X} \text { ) }\end{array}$ & mônade & $\begin{array}{l}\text { isopolar- } \\
\text { radiossimétrico }\end{array}$ & $\begin{array}{c}\mathrm{P}=33 \times \mathrm{E}=28 \\
\mathrm{P} / \mathrm{E}=1.17 ; \mathrm{Ex}=5.5 \\
\mathrm{Epa}=4.5 ; \mathrm{Epl}=2\end{array}$ & tricolporado & equinada & subprolato & \\
\hline \multicolumn{8}{|l|}{ Bignoniaceae } \\
\hline $\begin{array}{l}\text { Tipo } 35 \text { Bignoniaceae } \\
\text { (Figura 3Y) }\end{array}$ & mônade & $\begin{array}{l}\text { isopolar- } \\
\text { radiossimétrico }\end{array}$ & $\begin{array}{c}\mathrm{P}=31 \times \mathrm{E}=30 \\
\mathrm{P} / \mathrm{E}=1.03 ; \mathrm{Ex}=2\end{array}$ & tricolpado & microrreticulada & $\begin{array}{l}\text { prolato } \\
\text { esferoidal }\end{array}$ & \\
\hline \multicolumn{8}{|l|}{ Bignoniaceae } \\
\hline $\begin{array}{l}\text { Tipo } 36 \text { Tabebuia } \\
\text { Gomes ex DC., } 1838 \text { (Figura } \\
\text { 4A) }\end{array}$ & mônade & $\begin{array}{l}\text { isopolar- } \\
\text { radiossimétrico }\end{array}$ & $\begin{array}{c}\mathrm{P}=34 \times \mathrm{E}=21 ; \\
\mathrm{P} / \mathrm{E}=1.61 ; \mathrm{Ex}=1\end{array}$ & tricolporado & microrreticulada & prolato & \\
\hline $\begin{array}{l}\text { Tipo } 37 \text { cf. Arrabidaea } \\
\text { DC., } 1838 \text { (Figura 4B) }\end{array}$ & mônade & $\begin{array}{l}\text { isopolar- } \\
\text { radiossimétrico }\end{array}$ & $\begin{array}{c}\mathrm{P}=36 \times \mathrm{E}=23 ; \\
\mathrm{P} / \mathrm{E}=1.56 ; \mathrm{Ex}=<2\end{array}$ & tricolporado & $\begin{array}{c}\text { reticulo- } \\
\text { baculada, } \\
\text { homobrocada }\end{array}$ & prolato & \\
\hline $\begin{array}{l}\text { Tipo } 38 \text { Adenocalymma } \\
\text { Mart. \& Meisn., } 1840 \text { (Figura } \\
\text { 4C) }\end{array}$ & mônade & $\begin{array}{l}\text { isopolar- } \\
\text { radiossimétrico }\end{array}$ & $\begin{array}{c}\mathrm{P}=71 \times \mathrm{E}=68 \\
\quad \mathrm{Ex}=4.5\end{array}$ & inaperturado & $\begin{array}{l}\text { reticulo- } \\
\text { baculada, } \\
\text { homobrocada }\end{array}$ & circular & \\
\hline \multicolumn{8}{|l|}{ Boraginaceae } \\
\hline $\begin{array}{l}\text { Tipo } 39 \text { cf. Cordia } \\
\text { L., } 1753 \text { (Figura 4D) }\end{array}$ & mônade & $\begin{array}{l}\text { isopolar- } \\
\text { radiossimétrico }\end{array}$ & $\begin{array}{c}\mathrm{P}=50 \times \mathrm{E}=52 \\
\mathrm{Ex}=<3\end{array}$ & inaperturado & $\begin{array}{l}\text { reticulo- } \\
\text { equinada }\end{array}$ & circular & \\
\hline \multicolumn{8}{|l|}{ Brassicaceae } \\
\hline $\begin{array}{l}\text { Tipo } 40 \text { Capparis } \\
\text { L., } 1753 \text { (Figura 4E) }\end{array}$ & mônade & $\begin{array}{l}\text { isopolar- } \\
\text { radiossimétrico }\end{array}$ & $\begin{array}{c}\mathrm{P}=24 \times \mathrm{E}=19 ; \\
\mathrm{P} / \mathrm{E}=1.26 ; \mathrm{Ex}=<1\end{array}$ & tricolporado & reticulada & subprolato & $\begin{array}{c}\text { endoabertura lalongada; } \\
\text { poros circulares }\end{array}$ \\
\hline \multicolumn{8}{|l|}{ Cannabaceae } \\
\hline $\begin{array}{l}\text { Tipo } 41 \text { Celtis } \\
\text { L., } 1753 \text { (Figura 4F) }\end{array}$ & mônade & $\begin{array}{l}\text { isopolar- } \\
\text { radiossimétrico }\end{array}$ & $\begin{array}{c}\mathrm{P}=20 \times \mathrm{E}=19 \\
\quad \mathrm{Ex}=>1\end{array}$ & 3-4-porado & escabrada & circular & \\
\hline $\begin{array}{l}\text { Tipo } 42 \text { Trema } \\
\text { Lour., } 1790 \text { (Figura 4G) }\end{array}$ & mônade & $\begin{array}{l}\text { isopolar- } \\
\text { radiossimétrico }\end{array}$ & $P=21 \times E=21 ; E x=1$ & diporado & escabrada & circular & \\
\hline \multicolumn{8}{|l|}{ Cactaceae } \\
\hline $\begin{array}{l}\text { Tipo } 43 \text { Cactaceae } \\
\text { (Figura } 4 \mathrm{H} \text { ) }\end{array}$ & mônade & $\begin{array}{l}\text { isopolar- } \\
\text { radiossimétrico }\end{array}$ & $\begin{array}{c}\mathrm{P}=48 \times \mathrm{E}=43 \\
\mathrm{Ex}=<1\end{array}$ & tricolpado & equinada & circular & $\begin{array}{c}\text { endoabertura lalongada; } \\
\text { poros circulares }\end{array}$ \\
\hline \multicolumn{8}{|l|}{ Celastraceae } \\
\hline $\begin{array}{l}\text { Tipo } 44 \text { cf. Maytenus } \\
\text { Molina, } 1782 \text { (Figura 4I) }\end{array}$ & mônade & $\begin{array}{l}\text { isopolar- } \\
\text { radiossimétrico }\end{array}$ & $\begin{array}{c}\mathrm{P}=23 \times \mathrm{E}=19 ; \\
\mathrm{P} / \mathrm{E}=1.21 ; \mathrm{Ex}=1.5\end{array}$ & tricolporado & $\begin{array}{c}\text { reticulada- } \\
\text { homobrocada }\end{array}$ & subprolato & \\
\hline
\end{tabular}


Apêndice 1. Continua.

\begin{tabular}{|c|c|c|c|c|c|c|c|}
\hline Tipos polínicos & $\begin{array}{l}\text { Unidade } \\
\text { polínica }\end{array}$ & $\begin{array}{l}\text { Polaridade/ } \\
\text { Simetria }\end{array}$ & Medidas $(\mu \mathrm{m})$ & $\begin{array}{c}\text { NPC } \\
\text { aberturas/ } \\
\text { Cicatriz }\end{array}$ & Exina/ Perina & $\begin{array}{l}\text { Forma/ } \\
\text { Âmbito }\end{array}$ & Observações \\
\hline \multicolumn{8}{|l|}{ Clusiaceae } \\
\hline $\begin{array}{l}\text { Tipo } 45 \text { cf. Garcinia } \\
\text { L., } 1753 \text { (Figura 4J) }\end{array}$ & mônade & $\begin{array}{l}\text { isopolar- } \\
\text { radiossimétrico }\end{array}$ & $\begin{array}{c}\mathrm{P}=33 \times \mathrm{E}=14 \\
\mathrm{P} / \mathrm{E}=2.35 ; \mathrm{Ex}=<1\end{array}$ & tricolporado & escabrada & perprolato & poros elípticos \\
\hline $\begin{array}{l}\text { Tipo } 46 \text { cf. Clusia } \\
\text { L., } 1753 \text { (Figura 4K) }\end{array}$ & mônade & $\begin{array}{l}\text { isopolar- } \\
\text { radiossimétrico }\end{array}$ & $\begin{array}{c}\mathrm{P}=21 \times \mathrm{E}=20 \\
\quad \mathrm{Ex}=<1\end{array}$ & triporado & rugulada & circular & \\
\hline \multicolumn{8}{|l|}{ Combretaceae } \\
\hline $\begin{array}{l}\text { Tipo } 47 \text { Combretaceae } \\
\text { (Figura 4L) }\end{array}$ & mônade & $\begin{array}{l}\text { isopolar- } \\
\text { radiossimétrico }\end{array}$ & $\begin{array}{c}\mathrm{P}=19 \times \mathrm{E}=11 \\
\mathrm{P} / \mathrm{E}=1.72 ; \mathrm{Ex}=<1\end{array}$ & heterocolpado & $\begin{array}{l}\text { psilada a } \\
\text { escabrada }\end{array}$ & prolato & \\
\hline $\begin{array}{l}\text { Tipo } 48 \text { cf. Laguncularia } \\
\text { C.F. Gaertn., } 1791 \text { (Figura 4M) }\end{array}$ & mônade & $\begin{array}{l}\text { isopolar- } \\
\text { radiossimétrico }\end{array}$ & $\begin{array}{c}\mathrm{P}=15 \times \mathrm{E}=14 \\
\mathrm{P} / \mathrm{E}=1.07 ; \mathrm{Ex}=<1\end{array}$ & tricolporado & reticulada & oblato & endoabertura elíptica \\
\hline \multicolumn{8}{|l|}{ Connaraceae } \\
\hline $\begin{array}{l}\text { Tipo } 49 \text { Connarus } \\
\text { L., } 1753 \text { (Figura 4N) }\end{array}$ & mônade & $\begin{array}{l}\text { isopolar- } \\
\text { radiossimétrico }\end{array}$ & $\begin{array}{l}\mathrm{P}=20 \times \mathrm{E}=10 \\
\mathrm{P} / \mathrm{E}=2 ; \mathrm{Ex}=<1\end{array}$ & tricolporado & $\begin{array}{l}\text { reticulada- } \\
\text { homobrocada }\end{array}$ & prolato & \\
\hline \multicolumn{8}{|l|}{ Convolvulaceae } \\
\hline $\begin{array}{l}\text { Tipo } 50 \text { Convolvulaceae } \\
\text { (Figura 4O) }\end{array}$ & mônade & $\begin{array}{l}\text { isopolar- } \\
\text { radiossimétrico }\end{array}$ & $P=61 \times E=57 ; E x=3$ & tricolpado & baculada & circular & \\
\hline \multicolumn{8}{|l|}{ Elaeocarpaceae } \\
\hline $\begin{array}{l}\text { Tipo } 51 \text { Sloanea } \\
\text { L., } 1753 \text { (Figura 4P) }\end{array}$ & mônade & $\begin{array}{l}\text { isopolar- } \\
\text { radiossimétrico }\end{array}$ & $P=17 \times E=13 ; E x=1$ & tricolporado & escabrada & subprolato & \\
\hline \multicolumn{8}{|l|}{ Ericaceae } \\
\hline $\begin{array}{l}\text { Tipo } 52 \text { Ericaceae } \\
\text { (Figura 4Q) }\end{array}$ & tétrade & $\begin{array}{l}\text { isopolar- } \\
\text { radiossimétrico }\end{array}$ & $\begin{array}{c}\mathrm{P}=20 \times \mathrm{E}=19 \\
\quad \mathrm{Ex}=1.5\end{array}$ & trizonohemicolpado & psilada & circular & \\
\hline \multicolumn{8}{|l|}{ Erythroxylaceae } \\
\hline $\begin{array}{l}\text { Tipo } 53 \text { Erythroxylum } \\
\text { P.Browne, } 1756 \text { (Figura 4R) }\end{array}$ & mônade & $\begin{array}{l}\text { isopolar- } \\
\text { radiossimétrico }\end{array}$ & $\begin{aligned} \mathrm{P} & =47 \times \mathrm{E}=37 \\
\mathrm{P} / \mathrm{E} & =1.27 ; \mathrm{Ex}=3.5\end{aligned}$ & tricolporado & $\begin{array}{l}\text { reticulada- } \\
\text { homobrocada }\end{array}$ & subprolato & \\
\hline \multicolumn{8}{|l|}{ Euphorbiaceae } \\
\hline $\begin{array}{l}\text { Tipo } 54 \text { Actinostemon } \\
\text { Mart. ex Klotzsch, } 1841 \text { (Figura 4S) }\end{array}$ & mônade & $\begin{array}{l}\text { isopolar- } \\
\text { radiossimétrico }\end{array}$ & $\begin{aligned} \mathrm{P} & =44 \times \mathrm{E}=27 \\
\mathrm{P} / \mathrm{E} & =1.62 ; \mathrm{Ex}=1.5\end{aligned}$ & tricolporado & reticulada & prolato & poros lolongados \\
\hline $\begin{array}{l}\text { Tipo } 55 \text { Alchornea } \\
\text { Sw., } 1788 \text { (Figura 4T) }\end{array}$ & mônade & $\begin{array}{l}\text { isopolar- } \\
\text { radiossimétrico }\end{array}$ & $\begin{array}{c}\mathrm{P}=30 \times \mathrm{E}=30 ; \mathrm{Enc}= \\
5 ; \mathrm{Enl}=5 ; \mathrm{Ex}=2.5\end{array}$ & tricolporado & $\begin{array}{l}\text { psilada e } \\
\text { rugulada }\end{array}$ & subtriangular & $\begin{array}{l}\text { colpos longos e estreitos, } \\
\text { cobertos por opérculo; } \\
\text { endoaberturas lolongadas }\end{array}$ \\
\hline $\begin{array}{l}\text { Tipo } 56 \text { Croton } \\
\text { L., } 1753 \text { (Figura 4U) }\end{array}$ & mônade & $\begin{array}{l}\text { apolar- } \\
\text { radiossimétrico }\end{array}$ & $D=73 \times 73 ; E x=6$ & inaperturado & clavada & circular & padrão crotonóide \\
\hline $\begin{array}{l}\text { Tipo } 57 \text { Dalechampia } \\
\text { L., } 1753 \text { (Figura 4V) }\end{array}$ & mônade & $\begin{array}{l}\text { isopolar- } \\
\text { radiossimétrico }\end{array}$ & $\begin{array}{c}\mathrm{P}=57 \times \mathrm{E}=56 \\
\mathrm{P} / \mathrm{E}=1.01 ; \mathrm{Ex}=3.5\end{array}$ & tricolporado & $\begin{array}{c}\text { reticulada- } \\
\text { heterobrocada }\end{array}$ & esferoidal & $\begin{array}{c}\text { endoaberturas lalongadas, } \\
\text { endocinguladas }\end{array}$ \\
\hline $\begin{array}{l}\text { Tipo } 58 \text { Euphorbiaceae } \\
\text { (Figura 4W) }\end{array}$ & mônade & $\begin{array}{l}\text { isopolar- } \\
\text { radiossimétrico }\end{array}$ & $\begin{aligned} \mathrm{P} & =40 \times \mathrm{E}=22 \\
\mathrm{P} / \mathrm{E} & =1.81 ; \mathrm{Ex}=3.5\end{aligned}$ & tricolporado & reticulada & prolato & poros circulares \\
\hline $\begin{array}{l}\text { Tipo } 59 \text { Pachystroma } \\
\text { Müll.Arg., } 1865 \text { (Figura 5A) }\end{array}$ & mônade & $\begin{array}{l}\text { isopolar- } \\
\text { radiossimétrico }\end{array}$ & $\begin{array}{c}\mathrm{P}=48 \times \mathrm{E}=34 \\
\mathrm{P} / \mathrm{E}=1.41 ; \mathrm{Ex}=3\end{array}$ & tricolporado & $\begin{array}{c}\text { reticulada- } \\
\text { heterobrocada }\end{array}$ & prolato & poros elípticos \\
\hline $\begin{array}{l}\text { Tipo } 60 \text { Sapium } \\
\text { P.Browne, } 1756 \text { (Figura 5B) }\end{array}$ & mônade & $\begin{array}{l}\text { isopolar- } \\
\text { radiossimétrico }\end{array}$ & $\begin{array}{c}\mathrm{P}=37 \times \mathrm{E}=25 \\
\mathrm{P} / \mathrm{E}=1.48 ; \mathrm{Ex}=3\end{array}$ & trizonocolporado & baculada & prolato & \\
\hline $\begin{array}{l}\text { Tipo } 61 \text { Sebastiania } \\
\text { Bertol., } 1822 \text { (Figura 5C) }\end{array}$ & mônade & $\begin{array}{l}\text { isopolar- } \\
\text { radiossimétrico }\end{array}$ & $\begin{array}{c}\mathrm{P}=31 \times \mathrm{E}=24 ; \\
\mathrm{P} / \mathrm{E}=1.29 ; \mathrm{Ex}=3\end{array}$ & tricolporado & microrreticulada & subprolato & $\begin{array}{l}\text { colpos longos; } \\
\text { endoabertura lalongada }\end{array}$ \\
\hline \multicolumn{8}{|l|}{ Fabaceae-Caesalpinioideae } \\
\hline $\begin{array}{l}\text { Tipo } 62 \text { Fabaceae- } \\
\text { Caesalpinioideae } 1 \text { (Figura 5D) }\end{array}$ & mônade & $\begin{array}{c}\text { isopolar- } \\
\text { radiossimétrico }\end{array}$ & $\begin{array}{c}\mathrm{P}=43 \times \mathrm{E}=24 \\
\mathrm{P} / \mathrm{E}=1.79 ; \mathrm{Ex}=<2.5\end{array}$ & tricolporado & reticulada & prolato & colpos longos \\
\hline $\begin{array}{l}\text { Tipo } 63 \text { Fabaceae- } \\
\text { Caesalpinioideae } 2 \text { (Figura 5E) }\end{array}$ & mônade & $\begin{array}{l}\text { isopolar- } \\
\text { radiossimétrico }\end{array}$ & $\begin{array}{c}\mathrm{P}=30 \times \mathrm{E}=22 \\
\mathrm{P} / \mathrm{E}=1.36 ; \mathrm{Ex}=<2\end{array}$ & tricolporado & microrreticulada & prolato & colpos longos \\
\hline $\begin{array}{l}\text { Tipo } 64 \text { Bauhinia } \\
\text { L., } 1753 \text { (Figura 5F) }\end{array}$ & mônade & $\begin{array}{l}\text { isopolar- } \\
\text { radiossimétrico }\end{array}$ & $\begin{array}{c}\mathrm{P}=50 \times \mathrm{E}=47 \\
\mathrm{Ex}=<5\end{array}$ & sincolporado & $\begin{array}{l}\text { escabrada com } \\
\text { verrugas }\end{array}$ & circular & colpos longos \\
\hline $\begin{array}{l}\text { Tipo } 65 \text { Caesalpinia } \\
\text { L., } 1753 \text { (Figura 5G) }\end{array}$ & mônade & $\begin{array}{l}\text { isopolar- } \\
\text { radiossimétrico }\end{array}$ & $\begin{array}{c}\mathrm{P}=55 \times \mathrm{E}=40 \\
\mathrm{P} / \mathrm{E}=1.37 ; \mathrm{Ex}=>3\end{array}$ & tricolporado & reticulada & prolato & poros circulares \\
\hline $\begin{array}{l}\text { Tipo } 66 \text { Copaifera } \\
\text { L., } 1762 \text { (Figura } 5 \mathrm{H} \text { ) }\end{array}$ & mônade & $\begin{array}{l}\text { isopolar- } \\
\text { radiossimétrico }\end{array}$ & $\begin{array}{c}\mathrm{P}=35 \times \mathrm{E}=31 \\
\mathrm{Ex}=>2\end{array}$ & tricolporado & microrreticulada & triangular & \\
\hline $\begin{array}{l}\text { Tipo } 67 \text { cf. Melanoxylon } \\
\text { Schott, } 1827 \text { (Figura 5I) }\end{array}$ & mônade & $\begin{array}{l}\text { isopolar- } \\
\text { radiossimétrico }\end{array}$ & $\begin{array}{c}\mathrm{P}=30 \times \mathrm{E}=29 \\
\mathrm{Ex}=>1\end{array}$ & tricolporado & $\begin{array}{l}\text { reticulada- } \\
\text { homobrocada }\end{array}$ & circular & colpos longos \\
\hline $\begin{array}{l}\text { Tipo } 68 \text { Peltogyne } \\
\text { Vogel, } 1837 \text { (Figura 5J) }\end{array}$ & mônade & $\begin{array}{l}\text { isopolar- } \\
\text { radiossimétrico }\end{array}$ & $P=35 \times E=34 ; E x=2$ & tricolporado & baculada & circular & poros circulares \\
\hline $\begin{array}{l}\text { Tipo } 69 \text { Pterogyne } \\
\text { Schrad. Ex Ness, } 1842 \text { (Figura 5K) }\end{array}$ & mônade & $\begin{array}{l}\text { isopolar- } \\
\text { radiossimétrico }\end{array}$ & $\begin{array}{l}\mathrm{P}=22 \times \mathrm{E}=11 ; \\
\mathrm{P} / \mathrm{E}=2 ; \mathrm{Ex}=<1\end{array}$ & tricolporado & psilada & prolato & poros circulares \\
\hline
\end{tabular}


Apêndice 1. Continua.

\begin{tabular}{|c|c|c|c|c|c|c|}
\hline Tipos polínicos & $\begin{array}{l}\text { Unidade } \\
\text { polínica }\end{array}$ & $\begin{array}{l}\text { Polaridade/ } \\
\text { Simetria }\end{array}$ & Medidas $(\mu \mathrm{m})$ & $\begin{array}{c}\text { NPC } \\
\text { aberturas/ } \\
\text { Cicatriz }\end{array}$ & Exina/ Perina & $\begin{array}{l}\text { Forma/ } \\
\text { Âmbito }\end{array}$ \\
\hline
\end{tabular}

Fabaceae-Mimosoideae

Tipo 70 Anadenanthera Speg., 1923 (Figura 5L)

Tipo 71 Mimosa

L., 1753 (Figura 5M)

Tipo 72 Piptadenia

Benth., 1840 (Figura 5N)

Fabaceae-Papilionoideae

Tipo 73 Fabaceae-Papilionoideae (Figura 5O)

Tipo 74 Acosmium Schott, 1827 (Figura 5P)

Tipo 75 Canavalia DC., 1763 (Figura 5Q)

Tipo 76 Dalbergia-Machaerium (Figura 5R)

Tipo 77 Lonchocarpus-Ormosia (Figura 5S)

Tipo 78 Platymiscium

Standl. \& L.O. Williams, 1950 mônade

(Figura 5T)

Tipo 79 Pterocarpus

Jacq., 1763 (Figura 5U)

Tipo 80 Swartzia

Schreb., 1791 (Figura 5V)

Tipo 81 Zollernia

Maximil. \& Nees, 1827 (Figura 5W)

Lamiaceae

Tipo 82 cf. Aegiphila

Jacq., 1767 (Figura 5X)

Lentibulariaceae

Tipo 83 Utricularia

L., 1753 (Figura 5Y)

Loranthaceae

Tipo 84 Struthanthus

Mart., 1830 (Figura 6A)

Lythraceae

Tipo 85 Cuphea

P.Browne, 1756 (Figura 6B)

Malpighiaceae

Tipo 86 Malpighiaceae 1

(Figura 6C)

Tipo 87 Malpighiaceae 2

(Figura 6D)

Tipo 88 Byrsonima

Rich. ex Kunth, 1822 (Figura 6E)

Malvaceae

Tipo 89 Abutilon

Mill., 1754 (Figura 6F)

Tipo 90 Ceiba

Mill., 1754 (Figura 6G)

Tipo 91 Hibiscus

L., 1753 (Figura 6H)

Tipo 92 Pseudobombax

Dugand, 1943 (Figura 6I)

Tipo 93 cf. Waltheria

L., 1753 (Figura 6J)

mônade

mônade

mônade

mônade

mônade

mônade

\begin{tabular}{|c|c|c|c|c|c|c|}
\hline políade & $\begin{array}{l}\text { isopolar- } \\
\text { radiossimétrico }\end{array}$ & $\begin{array}{c}\mathrm{EM}=34 \times \mathrm{Em}=31 \\
\mathrm{Ex}=>1\end{array}$ & 4-porado & verrucada & $\begin{array}{l}\text { elíptica a } \\
\text { circular }\end{array}$ & $\begin{array}{l}\text { políade composta de } 16 \\
\text { grãos de pólen }\end{array}$ \\
\hline políade & $\begin{array}{c}\text { isopolar- } \\
\text { radiossimétrico }\end{array}$ & $\begin{array}{c}E M=23 \times E m=16 \\
E x=>1\end{array}$ & inaperturado & escabrada & elíptica & $\begin{array}{l}\text { políade composta de } 4 \\
\text { grãos de pólen }\end{array}$ \\
\hline políade & $\begin{array}{l}\text { isopolar- } \\
\text { radiossimétrico }\end{array}$ & $\begin{array}{c}\mathrm{EM}=35 \times \mathrm{Em}=29 \\
\mathrm{Ex}=1\end{array}$ & 3-porado & escabrada & elíptica & $\begin{array}{l}\text { políade composta de } 12 \\
\text { grãos de pólen }\end{array}$ \\
\hline
\end{tabular}

mônade cisopolar- $\quad \mathrm{P}=25 \times \mathrm{E}=24 ; \quad$ tricolporado reticulada circular

mônade $\begin{gathered}\text { isopolar- } \quad P=20 \times E=12 ; \\ \text { radiossimétrico } \mathrm{P} / \mathrm{E}=1.66 ; \mathrm{Ex}=<1\end{gathered}$ tricolporado reticulada prolato

mônade $\begin{gathered}\text { isopolar- } \\ \text { radiossimétrico }\end{gathered} \quad \begin{gathered}\mathrm{P}=35 \times \mathrm{E}=17 ; \\ \mathrm{Ex}=9\end{gathered}$ tricolporado rugulada triangular

$\quad \mathrm{Ex}=9$

mônade $\begin{array}{ccc}\text { isopolar- } & \mathrm{P} & =23 \times \mathrm{E}=18 ; \\ \text { radiossimétrico } & \mathrm{P} / \mathrm{E} & =1.27 ; \mathrm{Ex}=1.5\end{array}$ tricolporado $\begin{array}{rlr}\text { escabrada a } \\ \text { microrreticulada }\end{array}$ subprolato

colpos longos; poros circulares

isopolar- $\quad \mathrm{P}=28 \times \mathrm{E}=24$;

radiossimétrico $\mathrm{P} / \mathrm{E}=1.16 ; \mathrm{Ex}=<1.5$ tricolporado escabrada subprolato

poros circulares

isopolar- $\quad \mathrm{P}=21 \times \mathrm{E}=16$;

radiossimétrico $\mathrm{P} / \mathrm{E}=1.31 ; \mathrm{Ex}=<2$ tricolporado microrreticulada subprolato

poros elípticos

reticulada-

$\begin{array}{ccc}\text { isopolar- } & \mathrm{P}=26 \times \mathrm{E}=24 ; & \text { tricolporado } \\ \text { radiossimétrico } & \mathrm{Ex}=2.5\end{array}$

homobrocada

circular

isopolar-

$\mathrm{P}=23 \times \mathrm{E}=20$; tricolporado microrreticulo-

radiossimétrico $\mathrm{P} / \mathrm{E}=1.15 ; \mathrm{Ex}=<2$

baculada

subprolato

mônade isopolar- $\quad \mathrm{P}=29 \times \mathrm{E}=17$; $\quad$ tricolporado psilada a

escabrada

prolato

isopola

radiossimétrico

$\mathrm{P}=35 \times \mathrm{E}=34$

$\mathrm{Ex}=<3$

tricolporado

equinada

circular

espinhos curtos

$\begin{array}{lccc}\text { mônade } & \text { isopolar- } & \mathrm{P}=52 \times \mathrm{E}=50 ; & \text { 10-colporado } \\ \text { radiossimétrico } & \mathrm{Ex}=1.5 & \text { psilada } & \text { circular }\end{array}$

$\begin{array}{cccc}\text { isopolar- } & \mathrm{P}=25 \times \mathrm{E}=25 ; & \begin{array}{l}\text { tricolpado- } \\ \text { sincolpado }\end{array} & \text { psilada }\end{array} \quad$ triangular

mônade isopolar- $\quad \mathrm{P}=20 \times \mathrm{E}=20$;

radiossimétrico $\mathrm{Ex}=1$ sincolporado estriada triangular

mônade apolar-

$\mathrm{D}=37 \times 36 ; \mathrm{Ex}=>3 \quad 6-8$-porado

escabrada

circular

apolar-

assimétrico

$\mathrm{D}=57 \times 1 ; \mathrm{Ex}=2.5 \quad 10$-porado

rugulada

circular

isopolar-

$\mathrm{P}=13 \times \mathrm{E}=12$;

tricolporado

reticulada-

homobrocada

circular

poros lalongados

apolarassimétrico

equinada

circular

reticulada-

isopolar-

$\mathrm{D}=63 \times 65 ; \mathrm{Ex}=<9$ tricolporado

omobrocada

circular

colporado

equinada

circular

reticulada-

assimétrico $\quad \mathrm{Epa}=18 ; \mathrm{Epl}=7$

isopolar- $\quad \mathrm{P}=75 \times \mathrm{E}=70$;

radiossimétrico

$\mathrm{P}=75 \times \mathrm{E}=70 ;$
$\mathrm{Cl}=<9 ; \mathrm{Ex}=3$

periporado

brevi-

isopolar-

$\mathrm{P}=44 \times \mathrm{E}=43$;

tricolporado

homobrocada

triangular

estefano(4-5) reticulada-

colporado homobrocada circular 
Apêndice 1. Continua.

\begin{tabular}{|c|c|c|c|c|c|c|c|}
\hline Tipos polínicos & $\begin{array}{l}\text { Unidade } \\
\text { polínica }\end{array}$ & $\begin{array}{l}\text { Polaridade/ } \\
\text { Simetria }\end{array}$ & Medidas $(\mu \mathrm{m})$ & $\begin{array}{c}\text { NPC aberturas/ } \\
\text { Cicatriz }\end{array}$ & Exina/ Perina & $\begin{array}{l}\text { Forma/ } \\
\text { Âmbito }\end{array}$ & Observações \\
\hline \multicolumn{8}{|l|}{ Meliaceae } \\
\hline $\begin{array}{l}\text { Tipo } 94 \text { cf. Cedrela } \\
\text { (Figura } 6 \mathrm{~K} \text { ) }\end{array}$ & mônade & $\begin{array}{l}\text { isopolar- } \\
\text { radiossimétrico }\end{array}$ & $\begin{array}{c}\mathrm{P}=42 \times \mathrm{E}=28 \\
\mathrm{P} / \mathrm{E}=1.50 \\
\mathrm{Ex}=<1.5\end{array}$ & $\begin{array}{l}\text { estefano }(4-6) \\
\text { colporado }\end{array}$ & psilada & prolato & endoabertura circular \\
\hline $\begin{array}{l}\text { Tipo } 95 \text { cf. Trichilia } \\
\text { P.Browne, } 1756 \text { (Figura 6L) }\end{array}$ & mônade & $\begin{array}{c}\text { isopolar- } \\
\text { radiossimétrico }\end{array}$ & $\begin{aligned} \mathrm{P} & =26 \times \mathrm{E}=21 ; \\
\mathrm{P} / \mathrm{E} & =1.23 ; \mathrm{Ex}=<2\end{aligned}$ & $\begin{array}{c}\text { estefano (4-) } \\
\text { colporado }\end{array}$ & psilada & subprolato & endoabertura lalongada \\
\hline \multicolumn{8}{|l|}{ Moraceae } \\
\hline $\begin{array}{l}\text { Tipo } 96 \text { cf. Ficus } \\
\text { (Figura 6M) }\end{array}$ & mônade & $\begin{array}{l}\text { isopolar- } \\
\text { bilateral }\end{array}$ & $\begin{array}{c}\mathrm{P}=15 \times \mathrm{E}=10 \\
\mathrm{Ex}=<1\end{array}$ & diporado & psilada & elíptico & poros circulares \\
\hline \multicolumn{8}{|l|}{ Myrsinaceae } \\
\hline $\begin{array}{l}\text { Tipo } 97 \text { Rapanea } \\
\text { Aub., } 1775 \text { (Figura 6N) }\end{array}$ & mônade & $\begin{array}{l}\text { isopolar- } \\
\text { radiossimétrico }\end{array}$ & $\begin{array}{c}\mathrm{P}=26 \times \mathrm{E}=24 \\
\mathrm{Ex}=2\end{array}$ & $\begin{array}{l}\text { estefano (4) } \\
\text { colporado }\end{array}$ & perfurada & quadrangular & poros circulares \\
\hline \multicolumn{8}{|l|}{ Myrtaceae } \\
\hline $\begin{array}{l}\text { Tipo } 98 \text { Myrtaceae } 1 \\
\text { (Figura 6O) }\end{array}$ & mônade & $\begin{array}{c}\text { isopolar- } \\
\text { radiossimétrico }\end{array}$ & $\begin{array}{c}\mathrm{P}=17 \times \mathrm{x}=15 \\
\mathrm{Ex}=<1.5\end{array}$ & parassincolporado & granulada & triangular & $\begin{array}{l}\text { colpos longos, unidos aos } \\
\text { pólos }\end{array}$ \\
\hline $\begin{array}{l}\text { Tipo } 99 \text { Myrtaceae } 2 \\
\text { (Figura 6P) }\end{array}$ & mônade & $\begin{array}{l}\text { isopolar- } \\
\text { radiossimétrico }\end{array}$ & $\begin{array}{c}\mathrm{P}=17 \times \mathrm{E}=15 \\
\mathrm{Ex}=>1\end{array}$ & $\begin{array}{l}\text { 4-colporado- } \\
\text { sincolporado }\end{array}$ & escabrada & subtriangular & \\
\hline $\begin{array}{l}\text { Tipo } 100 \text { cf. Psidium } \\
\text { (Figura 6Q) }\end{array}$ & mônade & $\begin{array}{l}\text { isopolar- } \\
\text { radiossimétrico }\end{array}$ & $\begin{array}{c}\mathrm{P}=14 \times \mathrm{E}=13 ; \\
\mathrm{Ex}=1.5\end{array}$ & tricolporado & escabrada & triangular & $\begin{array}{l}\text { margem dos colpos com teto } \\
\text { contínuo, formando um } \\
\text { amplo vestíbulo }\end{array}$ \\
\hline \multicolumn{8}{|l|}{ Ochnaceae } \\
\hline $\begin{array}{l}\text { Tipo } 101 \text { Ouratea } \\
\text { Aubl., } 1775 \text { (Figura 6R) }\end{array}$ & mônade & $\begin{array}{c}\text { isopolar- } \\
\text { radiossimétrico }\end{array}$ & $\begin{array}{c}\mathrm{P}=18 \times \mathrm{E}=17 \\
\quad \mathrm{Ex}=1.5\end{array}$ & tricolporado & granulada & circular & \\
\hline \multicolumn{8}{|l|}{ Onagraceae } \\
\hline $\begin{array}{l}\text { Tipo } 102 \text { Ludwigia } \\
\text { L., } 1753 \text { (Figura 6S) }\end{array}$ & mônade & $\begin{array}{l}\text { isopolar- } \\
\text { radiossimétrico }\end{array}$ & $\begin{array}{c}\mathrm{P}=72 \times \mathrm{E}=61 \\
\mathrm{Ex}=4\end{array}$ & tricolporado & verrucada & subtriangular & poros circulares \\
\hline \multicolumn{8}{|l|}{ Passifloraceae } \\
\hline $\begin{array}{l}\text { Tipo } 103 \text { Passiflora L., } 1753 \\
\text { (Figura 7A) }\end{array}$ & mônade & $\begin{array}{c}\text { isopolar- } \\
\text { radiossimétrico }\end{array}$ & $\begin{array}{c}\mathrm{P}=70 \times \mathrm{E}=51 \\
\mathrm{Ex}=<5\end{array}$ & $\begin{array}{l}\text { estefano }(6-12) \\
\text { colpado }\end{array}$ & $\begin{array}{c}\text { reticulada- } \\
\text { heterobrocada }\end{array}$ & circular & colpos operculados \\
\hline \multicolumn{8}{|l|}{ Phyllanthaceae } \\
\hline $\begin{array}{l}\text { Tipo } 104 \text { Phyllanthaceae } \\
\text { (Figura 7B) }\end{array}$ & mônade & $\begin{array}{l}\text { isopolar- } \\
\text { radiossimétrico }\end{array}$ & $\begin{aligned} \mathrm{P} & =36 \times \mathrm{E}=25 ; \mathrm{P} / \mathrm{E} \\
& =1.44 ; \mathrm{Ex}=<2.5\end{aligned}$ & tricolporado & $\begin{array}{c}\text { reticulada- } \\
\text { homobrocada }\end{array}$ & prolato & $\begin{array}{l}\text { colpos longos; poros } \\
\text { circulares }\end{array}$ \\
\hline $\begin{array}{l}\text { Tipo } 105 \text { Hyeronima } \\
\text { Allemão, } 1848 \text { (Figura } 7 \mathrm{C})\end{array}$ & mônade & $\begin{array}{l}\text { isopolar- } \\
\text { radiossimétrico }\end{array}$ & $\begin{array}{c}\mathrm{P}=39 \times \mathrm{E}=19 ; \\
\mathrm{P} / \mathrm{E}=2.05 ; \mathrm{Ex}=2\end{array}$ & tricolporado & microrreticulada & perprolato & $\begin{array}{l}\text { poros lalongados; } \\
\text { endocingulado }\end{array}$ \\
\hline \multicolumn{8}{|l|}{ Polygalaceae } \\
\hline $\begin{array}{l}\text { Tipo } 106 \text { Polygala } \\
\text { L., } 1753 \text { (Figura 7D) } \\
\text { Polygonaceae }\end{array}$ & mônade & $\begin{array}{l}\text { isopolar- } \\
\text { radiossimétrico }\end{array}$ & $\begin{array}{c}\mathrm{P}=34 \times \mathrm{E}=22 ; \\
\mathrm{P} / \mathrm{E}=1.54 ; \mathrm{Ex}=<2\end{array}$ & $\begin{array}{l}\text { estefano }(8-10) \\
\text { colporado }\end{array}$ & psilada & prolato & poros circulares; zonorado \\
\hline $\begin{array}{l}\text { Tipo } 107 \text { Coccoloba } \\
\text { P.Browne, } 1756 \text { (Figura 7E) }\end{array}$ & mônade & $\begin{array}{l}\text { isopolar- } \\
\text { radiossimétrico }\end{array}$ & $\begin{array}{c}\mathrm{P}=33 \times \mathrm{E}=32 \\
\mathrm{Ex}=<2\end{array}$ & tricolporado & $\begin{array}{c}\text { reticulada- } \\
\text { homobrocada }\end{array}$ & circular & $\begin{array}{l}\text { colpos longos e estreitos, } \\
\text { suavizado nas margens, } \\
\text { endoaberturas lalongadas a } \\
\text { circulares }\end{array}$ \\
\hline $\begin{array}{l}\text { Tipo } 108 \text { Polygonum } \\
\text { L., } 1753 \text { (Figura } 7 F \text { ) }\end{array}$ & mônade & $\begin{array}{l}\text { isopolar- } \\
\text { radiossimétrico }\end{array}$ & $\begin{array}{c}\mathrm{P}=56 \times \mathrm{E}=52 \\
\mathrm{Ex}=<3.5\end{array}$ & periporado & $\begin{array}{l}\text { reticulo- } \\
\text { baculada, } \\
\text { heterobrocada }\end{array}$ & circular & \\
\hline \multicolumn{8}{|l|}{ Rhamnaceae } \\
\hline $\begin{array}{l}\text { Tipo } 109 \text { Rhamnaceae } \\
\text { (Figura } 7 \mathrm{G} \text { ) }\end{array}$ & mônade & $\begin{array}{l}\text { isopolar- } \\
\text { radiossimétrico }\end{array}$ & $\begin{array}{c}\mathrm{P}=24 \times \mathrm{E}=22 \\
\mathrm{Ex}=<1.5\end{array}$ & tricolporado & $\begin{array}{l}\text { psilada a } \\
\text { escabrada }\end{array}$ & triangular & \\
\hline \multicolumn{8}{|l|}{ Rhizophoraceae } \\
\hline $\begin{array}{l}\text { Tipo } 110 \text { Rhizophora } \\
\text { L., } 1753 \text { (Figura } 7 \mathrm{H} \text { ) }\end{array}$ & mônade & $\begin{array}{c}\text { isopolar- } \\
\text { radiossimétrico }\end{array}$ & $\begin{aligned} \mathrm{P} & =25 \times \mathrm{E}=23 ; \\
\mathrm{P} / \mathrm{E} & =1.23 ; \mathrm{Ex}=<2\end{aligned}$ & tricolporado & $\begin{array}{l}\text { microrreticulada- } \\
\text { homobrocada }\end{array}$ & & $\begin{array}{c}\text { colpos estreitos; } \\
\text { endoabertura lalongada }\end{array}$ \\
\hline \multicolumn{8}{|l|}{ Rubiaceae } \\
\hline $\begin{array}{l}\text { Tipo } 111 \text { Borreria } \\
\text { G.Mey., } 1818 \text { (Figura 7I) }\end{array}$ & mônade & $\begin{array}{l}\text { isopolar- } \\
\text { radiossimétrico }\end{array}$ & $\begin{array}{c}\mathrm{P}=27 \times \mathrm{E}=25 \\
\mathrm{Ex}=2\end{array}$ & $\begin{array}{l}\text { estefano }(5-) \\
\text { colporado }\end{array}$ & $\begin{array}{l}\text { granulo- } \\
\text { baculada }\end{array}$ & circular & \\
\hline $\begin{array}{l}\text { Tipo } 112 \text { Psychotria } \\
\text { L., } 1753 \text { (Figura } 7 J \text { ) }\end{array}$ & mônade & $\begin{array}{l}\text { isopolar- } \\
\text { radiossimétrico }\end{array}$ & $\begin{aligned} \mathrm{P} & =26 \times \mathrm{E}=21 ; \\
\mathrm{P} / \mathrm{E} & =1.23 ; \mathrm{Ex}=<2\end{aligned}$ & tricolporado & $\begin{array}{l}\text { reticulo- } \\
\text { baculada }\end{array}$ & suprolato & \\
\hline \multicolumn{8}{|l|}{ Rutaceae } \\
\hline $\begin{array}{l}\text { Tipo } 113 \text { Zanthoxylum } \\
\text { L., } 1753 \text { (Figura } 7 \mathrm{~K} \text { ) }\end{array}$ & mônade & $\begin{array}{l}\text { isopolar- } \\
\text { radiossimétrico }\end{array}$ & $\begin{array}{c}P=17 \times E=14 \\
E x=2\end{array}$ & tricolporado & $\begin{array}{c}\text { reticulada- } \\
\text { homobrocada }\end{array}$ & circular & \\
\hline \multicolumn{8}{|l|}{ Sapindaceae } \\
\hline $\begin{array}{l}\text { Tipo } 114 \text { Allophylus } \\
\text { Gled., } 1764 \text { (Figura 7L) }\end{array}$ & mônade & $\begin{array}{c}\text { isopolar- } \\
\text { radiossimétrico }\end{array}$ & $\begin{array}{c}\mathrm{P}=53 \times \mathrm{E}=40 \\
\mathrm{Ex}=<2\end{array}$ & 4-porado & $\begin{array}{c}\text { reticulada- } \\
\text { homobrocada }\end{array}$ & triangular & \\
\hline
\end{tabular}


Apêndice 1. Continua.

\begin{tabular}{|c|c|c|c|c|c|c|c|}
\hline Tipos polínicos & $\begin{array}{l}\text { Unidade } \\
\text { polínica }\end{array}$ & $\begin{array}{l}\text { Polaridade/ } \\
\text { Simetria }\end{array}$ & Medidas $(\mu \mathrm{m})$ & $\begin{array}{c}\text { NPC } \\
\text { aberturas/ } \\
\text { Cicatriz }\end{array}$ & Exina/ Perina & $\begin{array}{l}\text { Forma/ } \\
\text { Âmbito }\end{array}$ & Observações \\
\hline \multicolumn{8}{|l|}{ Phyllanthaceae } \\
\hline $\begin{array}{l}\text { Tipo } 115 \text { Paullinia } \\
\text { L., } 1753 \text { (Figura } 7 \mathrm{M} \text { ) }\end{array}$ & mônade & $\begin{array}{l}\text { isopolar- } \\
\text { radiossimétrico }\end{array}$ & $\begin{array}{c}\mathrm{P}=28 \times \mathrm{E}=26 \\
\mathrm{Ex}=<1.5\end{array}$ & triporado & $\begin{array}{c}\text { reticulada- } \\
\text { homobrocada }\end{array}$ & triangular & $\begin{array}{c}\text { poros anulados; presença } \\
\text { de hemicolpos }\end{array}$ \\
\hline $\begin{array}{l}\text { Tipo } 116 \text { Serjania } \\
\text { Vell., } 1829 \text { (Figura } 7 \mathrm{~N} \text { ) }\end{array}$ & mônade & $\begin{array}{l}\text { isopolar- } \\
\text { radiossimétrico }\end{array}$ & $\begin{array}{c}\mathrm{P}=32 \times \mathrm{E}=32 \\
\mathrm{Ex}=<1.5\end{array}$ & $\begin{array}{l}\text { tricolporado, } \\
\text { sincolporado }\end{array}$ & perfurada & triangular & colpos longos e estreitos \\
\hline \multicolumn{8}{|l|}{ Solanaceae } \\
\hline $\begin{array}{l}\text { Tipo } 117 \text { Solanum } \\
\text { L., } 1753 \text { (Figura } 70 \text { ) }\end{array}$ & mônade & $\begin{array}{l}\text { isopolar- } \\
\text { radiossimétrico }\end{array}$ & $\begin{array}{c}\mathrm{P}=21 \times \mathrm{E}=18 \\
\mathrm{Ex}=<1.5\end{array}$ & tricolporado & psilada & circular & poros lalongados \\
\hline \multicolumn{8}{|l|}{ Urticaceae } \\
\hline $\begin{array}{l}\text { Tipo } 118 \text { cf. Cecropia } \\
\text { Loefl., } 1758 \text { (Figura 7P) }\end{array}$ & mônade & $\begin{array}{l}\text { isopolar- } \\
\text { bilateral }\end{array}$ & $\begin{aligned} \mathrm{P} & =13 \times \mathrm{E}=8 ; \\
\mathrm{P} / \mathrm{E} & =1.62 ; \mathrm{Ex}=<1\end{aligned}$ & diporado & $\begin{array}{l}\text { psilada a } \\
\text { granulada }\end{array}$ & prolato & \\
\hline \multicolumn{8}{|l|}{ Verbenaceae } \\
\hline $\begin{array}{l}\text { Tipo } 119 \text { cf. Lantana } \\
\text { L., } 1753 \text { (Figura 7Q) }\end{array}$ & mônade & $\begin{array}{l}\text { isopolar- } \\
\text { radiossimétrico }\end{array}$ & $\begin{array}{c}\mathrm{P}=30 \times \mathrm{E}=29 \\
\mathrm{Ex}=<2\end{array}$ & tricolporado & granulada & circular & \\
\hline
\end{tabular}

